\title{
Calabi-Yau compactifications of non-supersymmetric heterotic string theory
}

\author{
Michael Blaszczyk, ${ }^{a}$ Stefan Groot Nibbelink, ${ }^{b}$ Orestis Loukas ${ }^{b, c}$ and Fabian Ruehle ${ }^{d}$ \\ ${ }^{a}$ PRISMA Cluster of Excellence $\&$ Institut für Physik (WA THEP), \\ Johannes-Gutenberg-Universität, \\ 55099 Mainz, Germany \\ ${ }^{b}$ Arnold Sommerfeld Center for Theoretical Physics, Ludwig-Maximilians-Universität München, \\ 80333 München, Germany \\ ${ }^{c}$ School of Electrical and Computer Engineering, National Technical University of Athens, \\ Zografou Campus, GR-15780 Athens, Greece \\ ${ }^{d}$ Deutsches Elektronen-Synchrotron DESY, \\ Notkestrasse 85, 22607 Hamburg, Germany \\ E-mail: blaszcz@uni-mainz.de, Groot.Nibbelink@physik.uni-muenchen.de, \\ 0.Loukas@physik.uni-muenchen.de, fabian.ruehle@desy.de
}

ABStRaCt: Phenomenological explorations of heterotic strings have conventionally focused primarily on the $\mathrm{E}_{8} \times \mathrm{E}_{8}$ theory. We consider smooth compactifications of all three ten-dimensional heterotic theories to exhibit the many similarities between the non-supersymmetric $\mathrm{SO}(16) \times \mathrm{SO}(16)$ theory and the related supersymmetric $\mathrm{E}_{8} \times \mathrm{E}_{8}$ and $\mathrm{SO}(32)$ theories. In particular, we exploit these similarities to determine the bosonic and fermionic spectra of Calabi-Yau compactifications with line bundles of the nonsupersymmetric string. We use elements of four-dimensional supersymmetric effective field theory to characterize the non-supersymmetric action at leading order and determine the Green-Schwarz induced axion couplings. Using these methods we construct a non-supersymmetric Standard Model(SM)-like theory. In addition, we show that it is possible to obtain SM-like models from the standard embedding using at least an order four Wilson line. Finally, we make a proposal of the states that live on five-branes in the $\mathrm{SO}(16) \times \mathrm{SO}(16)$ theory and find under certain assumptions the surprising result that anomaly factorization only admits at most a single brane solution.

KEYWORDS: Strings and branes phenomenology

ARXIV EPRINT: 1507.06147 


\section{Contents}

1 Introduction and summary 1

2 Ten-dimensional heterotic strings $\quad 6$

$\begin{array}{lll}2.1 & \text { Effective ten-dimensional heterotic actions } & 7\end{array}$

2.2 Heterotic lattices 8

2.3 Non-supersymmetric twists 8

3 Smooth backgrounds $\quad 10$

3.1 Calabi-Yau manifolds 11

3.2 Line bundles on Calabi-Yaus 13

4 Spectra of smooth Calabi-Yau compactifications $\quad \mathbf{1 5}$

$\begin{array}{lll}4.1 & \text { Massless charged chiral 4D spectrum } & 15\end{array}$

$\begin{array}{ll}\text { 4.2 Dirac and Hirzebruch-Riemann-Roch indices } & 16\end{array}$

$\begin{array}{lll}4.3 & \text { Beyond the chiral spectrum } & 17\end{array}$

5 Effective theories in four dimensions $\quad \mathbf{1 9}$

5.1 Effective four-dimensional $\mathrm{N}=1$ actions for $\mathrm{E}_{8} \times \mathrm{E}_{8}$ and $\mathrm{SO}(32)$ compactifi$\begin{array}{lr}\text { cations } & 19\end{array}$

5.2 Elements of the effective $\mathrm{SO}(16) \times \mathrm{SO}(16)$ theory in four dimensions 22

6 Example of a smooth SM-like model $\quad 24$

7 Smooth SM-like models from the standard embedding 25

$8 \quad$ Heterotic five-branes in the $\mathrm{SO}(16) \times \mathrm{SO}(16)$ theory 27

A Traces $\quad 33$

B Line bundle description as $\mathrm{S}\left(\mathrm{U}(1)^{n+1}\right)$ bundles 35

B.1 A line bundle vector representation of $\mathrm{S}\left(\mathrm{U}(1)^{5}\right)$ bundles 36

B.2 A line bundle vector representation of $\mathrm{S}\left(\mathrm{U}(1)^{6}\right)$ bundles 36

\section{Introduction and summary}

One of the most frequently considered extensions of the Standard Model (SM) of Particle Physics is supersymmetry. This hypothetical symmetry assigns to each observed particle a supersymmetric partner which has identical properties except that their spins differ by $1 / 2$, i.e. it relates bosons to fermions and vice versa. One of the main goals of the LHC accelerator at CERN is to observe these superpartners. So far, there have been no hints for the existence of such states. 
One may wonder about the consequences for string theory if supersymmetry will not be found at the LHC or possible future accelerators. In light of the often stated claim that string theory predicts supersymmetry, this seems to be a doomsday scenario for string theory. However, the statement that string theory requires target space supersymmetry is simply false: as had been realized, essentially during the time that string theory was first considered as a unified framework of all particles and interactions, it is possible to construct consistent string theories without space-time supersymmetry. Minimal requirements for a consistent theory are modular invariance and the absence of anomalies and tachyons. A prime example of a non-supersymmetric string theory is the $\mathrm{SO}(16) \times \mathrm{SO}(16)$ string [1-3].

In the past decades various authors have considered non-supersymmetric string constructions [4-7]. Torus compactifications with Wilson lines of the $\mathrm{SO}(16) \times \mathrm{SO}(16)$ theory have been studied in ref. [8, 9]. In addition, using a covariant lattice approach fourdimensional non-supersymmetric models were constructed by [10, 11]. Compactification on (a)symmetric orbifolds [2, 12-14] of the non-supersymmetric $\mathrm{SO}(16) \times \mathrm{SO}(16)$ have been inspected in the papers [15-18]. Non-supersymmetric compactifications of the heterotic string have also been investigated using the free-fermionic string description [19-21] see e.g. the works [22-26]. Also in non-heterotic string context tachyon-free non-supersymmetric models have been constructed, for example in type-I [27-29], as type-II orientifolds [30-40] or rational conformal field theories [41, 42].

Last year various new investigations of the phenomenological potential of nonsupersymmetric compactifications of the heterotic string have appeared. In [43, 44] some of the authors of the current paper considered orbifold and smooth Calabi-Yau compactifications of the non-supersymmetric $\mathrm{SO}(16) \times \mathrm{SO}(16)$ string. It was argued that Calabi-Yau compactification of the $\mathrm{SO}(16) \times \mathrm{SO}(16)$ theory has particular phenomenological potential, since tachyons can be avoided to leading orders in the $\alpha^{\prime}$ and $g_{s}$ expansions. Moreover, it was shown that it is possible to obtain many tachyon-free orbifold models with spectra quite close to the Standard Model (SM).

Also certain half-flat geometries can be considered as non-supersymmetric backgrounds for the heterotic string [45]. In addition, semi-realistic models were constructed in the free-fermionic context in [46, 47], which implement the idea of having models that interpolate between supersymmetric and non-supersymmetric string constructions [22, 48-50]. For such models it is possible to compute more detailed phenomenological quantities like threshold corrections $[51,52]$.

Of course low-scale supersymmetry has been a very popular theory for physics beyond the SM for many years since it provides a solution to the hierarchy problem, helps with gauge coupling unification, and provides a natural dark matter candidate. While there are potential solutions known for the last two points (gauge coupling unification could be achived via Kaluza-Klein threshold corrections and string theory predicts many extra (hidden sector) particles which might serve as dark matter), a natural solution to the hierarchy problem in theories without supersymmetry is currently not known to the authors. This problem will have to be tackled in the future not only by string theory but by any theory beyond the SM if low-energy supersymmetry is not found. Even if it is found, current exclusion limits already point at a small hierarchy between the electroweak 


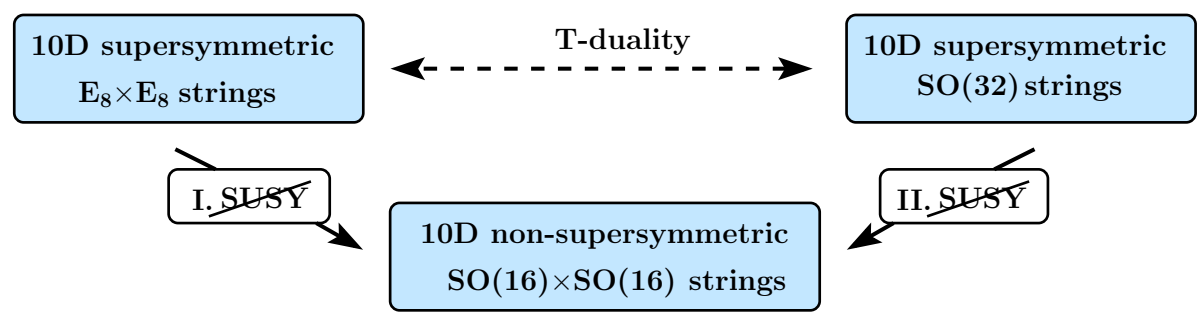

Figure 1. This figure depicts the relation between the three heterotic string theories in ten dimensions.

and the SUSY breaking scale that needs to be explained. These questions are, however, beyond the scope of this paper.

In this paper we lay out more theoretical methods to perform investigations of smooth compactifications of the non-supersymmetric $\mathrm{SO}(16) \times \mathrm{SO}(16)$ string. In order to do so, we often make use of the fact that the non-supersymmetric heterotic $\mathrm{SO}(16) \times \mathrm{SO}(16)$ string is closely related to the supersymmetric heterotic $\mathrm{E}_{8} \times \mathrm{E}_{8}$ and $\mathrm{SO}(32)$ strings: it is well-known that upon compactification on a circle with appropriate Wilson lines both supersymmetric heterotic strings become T-dual to each other. Moreover, the non-supersymmetric $\mathrm{SO}(16) \times \mathrm{SO}(16)$ theory can be obtained by supersymmetry-breaking twists acting on either the $\mathrm{E}_{8} \times \mathrm{E}_{8}$ or the $\mathrm{SO}(32)$ theories. These relations between the three heterotic theories in ten dimensions are indicated in figure 1. Interestingly, the full low energy spectrum of the $\mathrm{SO}(16) \times \mathrm{SO}(16)$ theory can be obtained by simple orbifold projections and interpreted as the combined untwisted sectors of the $\mathrm{E}_{8} \times \mathrm{E}_{8}$ and the $\mathrm{SO}(32)$ strings. As can be inferred from table 1 , the gravitational and gauge sectors can be obtained from either route. On the other hand, the chiral fermions in the spinor representation of the $\mathrm{SO}(16)$ factors come from the untwisted sector of the $\mathrm{E}_{8} \times \mathrm{E}_{8}$ string, while the chiral fermions in the bi-fundamental of $\mathrm{SO}(16) \times \mathrm{SO}(16)$ are part of its twisted sector w.r.t. the supersymmetry-breaking twist. In contrast, for the $\mathrm{SO}(32)$ theory the roles of untwisted and twisted chiral matter are precisely interchanged.

Next we investigate the effective four-dimensional theories that arise when we compactify one of the heterotic strings on Calabi-Yau geometries with (line) bundles. When we start from the non-supersymmetric $\mathrm{SO}(16) \times \mathrm{SO}(16)$ theory, there seems to be no need to consider string backgrounds that would themselves preserve some amount of supersymmetry. However, there are various reasons why insisting on Calabi-Yau geometries with holomorphic stable vector bundles is convenient:

From a worldsheet point of view, having a complex manifold with a holomorphic vector bundle means that one has an enhanced global $\mathrm{U}(1)_{R}$ symmetry so that the worldsheet theory has $(2,0)$ supersymmetry. This $\mathrm{U}(1)_{R}$ symmetry is non-anomalous precisely if the manifold is Calabi-Yau, see [53, 54]. Notice that, to leading order, these arguments do not depend on the global boundary conditions on the worldsheet, i.e. the spin-structures and hence they apply to each of the three heterotic theories.

Also from the target space Effective Field Theory (EFT) perspective, there are good reasons to consider supersymmetry-preserving compactifications of the $\mathrm{SO}(16) \times \mathrm{SO}(16)$ 


\begin{tabular}{|c|c|c|c|}
\hline Theory & Sector & Bosons & Fermions \\
\hline \multirow{2}{*}{$\begin{array}{c}\text { supersymmetric } \\
\mathrm{E}_{8} \times \mathrm{E}_{8} \\
\end{array}$} & gravity & metric, B-field, dilaton & gravitinos, dilatinos \\
\hline & gauge & $(248,1)+(1,248)$ gauge fields & $(248,1)+(1,248)$ gauginos \\
\hline \multirow{2}{*}{$\begin{array}{c}\text { supersymmetric } \\
\mathrm{SO}(32)\end{array}$} & gravity & metric, B-field, dilaton & gravitinos, dilatinos \\
\hline & gauge & 496 gauge fields & 496 gauginos \\
\hline \multirow{3}{*}{$\begin{array}{c}\text { non- } \\
\text { supersymmetric } \\
\mathrm{SO}(16) \times \mathrm{SO}(16)\end{array}$} & gravity & metric, B-field, dilaton & \\
\hline & gauge & $(120,1)+(1,120)$ gauge fields & \\
\hline & matter & & $\begin{array}{c}(\mathbf{1 2 8}, \mathbf{1})+(\mathbf{1}, \mathbf{1 2 8}) \text { spinors } \\
(\mathbf{1 6}, \mathbf{1 6}) \text { co-spinors }\end{array}$ \\
\hline
\end{tabular}

Table 1. This table gives the bosonic and fermionic spectra of the three consistent heterotic string theories with gauge groups $\mathrm{E}_{8} \times \mathrm{E}_{8}, \mathrm{SO}(32)$ and $\mathrm{SO}(16) \times \mathrm{SO}(16)$.

string. As stated above, it was shown in $[43,44]$ that such compactifications avoid tachyons to leading order in $g_{s}$ and $\alpha^{\prime}$. Moreover, the bosonic parts of the three heterotic tendimensional theories are identical up to their gauge groups. Consequently, the reduction of either of these theories on the same background leads to essentially identical EFTs in four dimensions. This means that the effective potential of $\mathrm{SO}(16) \times \mathrm{SO}(16)$ compactifications, relevant to characterize the physical vacuum, is characterized by the same quantities as compactifications of its supersymmetric cousins, at least to leading order. This shows that stable supersymmetric backgrounds (solutions to F- and D-term conditions at tree-level) also represent solutions to the field equations of the non-supersymmetric $\mathrm{SO}(16) \times \mathrm{SO}(16)$ theory. Also it turns out to be fruitful to employ concepts like the super- and Kähler potential to characterize its four-dimensional EFT.

All this suggests that many methods developed for Calabi-Yau compactifications can be used to obtain results for compactifications of the non-supersymmetric $\mathrm{SO}(16) \times \mathrm{SO}(16)$ theory as well. The supersymmetric heterotic theories [55-57], and in particular the $\mathrm{E}_{8} \times \mathrm{E}_{8}$ theory with non-Abelian bundles [58-60] or line bundles on orbifold resolutions [61-63] and Complete Intersection Calabi-Yau manifolds (CICYs) [64, 65], have been well-studied since the seminal paper [66]. (A technical side result that we derive in appendix B is how to translate the line bundle parameterization used in $[64,65]$ to the line bundle vector language of [61-63] following [67].) For the non-supersymmetric $\mathrm{SO}(16) \times \mathrm{SO}(16)$ there are in principle three ways to get access to the effective theory in four dimensions as depicted in figure 2.

The most direct route is indicated in the middle: one starts with the effective ten-dimensional non-supersymmetric $\mathrm{SO}(16) \times \mathrm{SO}(16)$ theory and compactifies it on a supersymmetry-preserving background. However, given that the supersymmetry-breaking twists do not act on the internal geometry at all, we can alternatively first compactify either of the supersymmetric theories on the same smooth background, and subsequently apply the supersymmetry-breaking twists. In other words figure 2 displays three alternative routes to obtain the compactifications of the $\mathrm{SO}(16) \times \mathrm{SO}(16)$ theory. This means that 


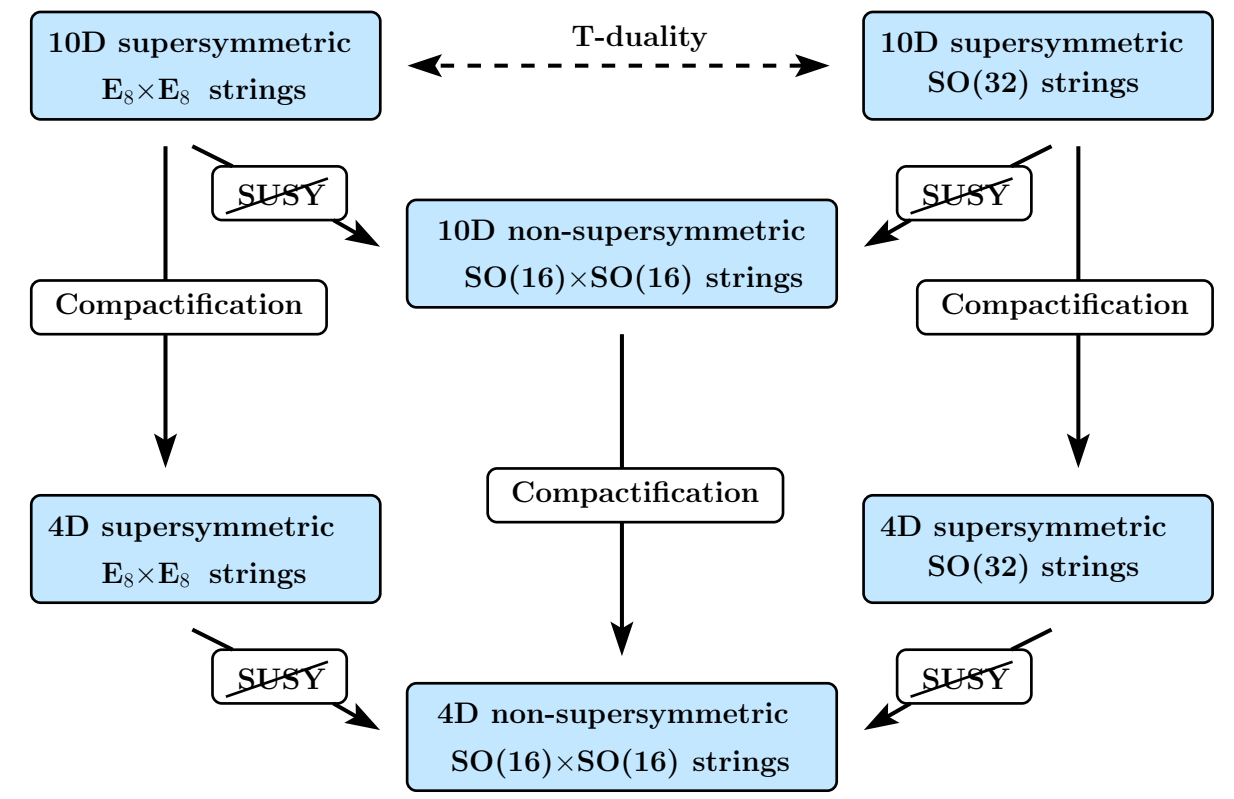

Figure 2. This double commutative diagram sketches the different routes that can be taken to determine the four-dimensional effective theory by compactification of the $\mathrm{SO}(16) \times \mathrm{SO}(16)$ theory on Calabi-Yau manifolds with holomorphic (line) bundles.

the spectrum of gauge fields and charged scalars obtained in Calabi-Yau compactifications of the $\mathrm{SO}(16) \times \mathrm{SO}(16)$ can be verified by compactifying either the $\mathrm{E}_{8} \times \mathrm{E}_{8}$ or $\mathrm{SO}(32)$ theory on the same background and subsequently applying the supersymmetry-breaking projections. Since the fermions of the $\mathrm{SO}(16) \times \mathrm{SO}(16)$ theory are both twisted and untwisted w.r.t. either supersymmetric string, one needs both the $\mathrm{E}_{8} \times \mathrm{E}_{8}$ and $\mathrm{SO}(32)$ compactification routes to determine the full charged chiral spectrum in four dimensions. As a consequence, many properties of compactifications of the non-supersymmetric $\mathrm{SO}(16) \times \mathrm{SO}(16)$ theory are closely related to results obtained in the past for compactifications of both supersymmetric heterotic strings.

This applies in particular to the Green-Schwarz anomaly cancellation mechanism. It is well-known that the Green-Schwarz mechanism is very important to obtain consistent string constructions both in ten and four dimensions since it ensures the cancellation of reducible anomalies. Moreover, it determines the couplings between model-dependent and independent axions and the gauge fields. For the Calabi-Yau compactifications of the supersymmetric heterotic theories these couplings were worked out in detail in [55-57]. In this paper we investigate the Green-Schwarz mechanism for smooth Calabi-Yau compactifications of the $\mathrm{SO}(16) \times \mathrm{SO}(16)$ string with line bundles. Using this we can perform model independent checks of anomaly cancellation for the chiral fermionic four-dimensional spectra obtained from these compactifications.

In this work we not only want to lay out the general framework of smooth compactifications of the non-supersymmetric $\mathrm{SO}(16) \times \mathrm{SO}(16)$ string, but we also want to show that it has true SM-like model building potential, as has been recently established for orbifold 
compactifications and free-fermionic constructions, as mentioned above. We argue that it is possible to obtain SM-like models from the standard embedding if the Calabi-Yau geometry admits at least an order four Wilson line. In addition, we present a particular SM-like model obtained on the smooth CICY with number 7862 (sometimes referred to as the tetra quadric) in the database [68-70] with line bundles. We construct a six generation non-supersymmetric SU(5) GUT, which upon using a freely acting Wilson line becomes a three generation SM-like model. In a follow-up work we will present some extensive model scans on various smooth geometries and the search for SM-like models. However, from our current analysis we can make one interesting observation concerning the Higgs sector of such compactifications: as we will explain in this paper, we can either have a single Higgs doublet together with a color triplet partner or we have at least one pair of vector-like Higgs-doublets.

Phenomenological model building on smooth compactifications of the $\mathrm{E}_{8} \times \mathrm{E}_{8}$ supergravity often makes use of the possibility of having five-branes. NS5-branes give an additional degree of freedom in model constructions since one does not have to satisfy the Bianchi identities strictly but only modulo effective curve classes. Effectiveness of these curves is crucial in order to guarantee that the same type of supersymmetry is preserved by the perturbative compactification and the non-perturbative NS5-brane sector. Contrary to the $\mathrm{E}_{8} \times \mathrm{E}_{8}$ theory, NS5-branes in the $\mathrm{SO}(32)$ context modify the spectrum charged under the perturbative gauge group. As far as we are aware five-branes in the non-supersymmetric $\mathrm{SO}(16) \times \mathrm{SO}(16)$ context have not been studied systematically in the literature. To gain some intuition for the possible properties of NS5-branes we take inspiration from the diagram in figure 2.

Since the fermions are the prime contributors to the anomalies, and since it is known which kind of NS5-branes the two supersymmetric heterotic theories require, we make an educated guess for the spectra on five-branes in the $\mathrm{SO}(16) \times \mathrm{SO}(16)$ theory: we take the spectra on the NS5-branes of the $\mathrm{E}_{8} \times \mathrm{E}_{8}$ or $\mathrm{SO}(32)$ theories and extend the supersymmetrybreaking twist to them. We find that this choice is essentially unique if we require that all irreducible gauge and gravitational anomalies cancel. If we want in addition to cancel all reducible anomalies via a generalized Green-Schwarz mechanism, e.g. involving scalars and tensors on the five-branes, we find (within the ansatz that we made for these couplings) that we can only achieve this with no NS5-branes or with a configuration with one $\mathrm{E}_{8} \times \mathrm{E}_{8}$-like and one $\mathrm{SO}(32)$-like NS5-brane.

\section{Ten-dimensional heterotic strings}

Conventionally, consistent string theories are characterized as string constructions that have low energy spectra which are free of anomalies and tachyons and have a modular invariant one-loop partition function. In ten dimensions there are three consistent heterotic string theories in this sense: the two that are most commonly studied, the $\mathrm{E}_{8} \times \mathrm{E}_{8}$ and $\mathrm{SO}(32)$ theories, are supersymmetric in target space. The third theory with gauge group $\mathrm{SO}(16) \times \mathrm{SO}(16)$ is non-supersymmetric. It is common to distinguish the three heterotic theories by their ten-dimensional gauge group, as will be done here as well. 


\subsection{Effective ten-dimensional heterotic actions}

Since we only concentrate on smooth compactifications, it is for most purposes sufficient to only consider the massless bosonic and fermionic spectrum in ten dimensions, which we give in table 1 for the three heterotic theories. Their effective target space descriptions are very similar. In the string frame their bosonic action is given by

$$
S_{10 D}=\frac{1}{2 \kappa_{10}^{2}} \int d^{10} x \sqrt{-\operatorname{det} G} e^{-2 \Phi}\left\{R\left(\omega_{+}\right)+4 \partial_{M} \Phi \partial^{M} \Phi-\frac{1}{2}\left|H_{3}\right|^{2}-\frac{\alpha^{\prime}}{4} \operatorname{tr}\left|F_{2}\right|^{2}\right\}
$$

where $\kappa_{10}^{2}=\frac{1}{2}(2 \pi)^{7}\left(\alpha^{\prime}\right)^{4}, \Phi$ is the dilaton and $G_{M N}$ the ten-dimensional metric. Its curvature scalar $R\left(\omega_{+}\right)$involves the spin-connection with torsion $\omega_{+}=\omega+\frac{1}{2} H . F_{2}$ denotes the non-Abelian gauge field strength. The field strength of the Kalb-Ramond field reads

$$
H_{3}=d B_{2}-\frac{\alpha^{\prime}}{4} X_{3}, \quad d X_{3}=X_{4}=\mathrm{TR} F_{2}^{2}-\mathrm{TR} R_{2}\left(\omega_{+}\right)^{2}
$$

Here TR denotes the trace in the fundamental ${ }^{1}$ (vector) representation of an $\mathrm{SO}(N)$ group. Since the adjoint representation of $\mathrm{E}_{8}$ is its fundamental we use $\mathrm{TR} F_{2}^{2}=\frac{1}{30} \operatorname{Tr} F_{2}^{2}$. In cases where we need to distinguish the two gauge factors of the $\mathrm{E}_{8} \times \mathrm{E}_{8}$ or $\mathrm{SO}(16) \times \mathrm{SO}(16)$ we denote their field strengths etc. by $F_{2}=\left(F_{2}^{\prime}, F_{2}^{\prime \prime}\right)$, e.g.: $\mathrm{TR} F_{2}^{2}=\mathrm{TR} F_{2}^{\prime 2}+\mathrm{TR} F_{2}^{\prime \prime 2}$. With the trace $\operatorname{Tr}$ we denote the trace over all charged Majorana-Weyl fermions. If we wish to make this more explicit, we denote by $\operatorname{Tr}_{\mathrm{E}_{8} \times \mathrm{E}_{8}}$ and $\operatorname{Tr}_{\mathrm{SO}(32)}$ the trace in the adjoint of $\mathrm{E}_{8} \times \mathrm{E}_{8}$ or $\mathrm{SO}(32)$ theories, respectively. While for the non-supersymmetric $\mathrm{SO}(16) \times \mathrm{SO}(16)$ we have

$$
\operatorname{Tr}_{\text {SUSY }} F_{2}^{p}=\left[\operatorname{tr}_{(\mathbf{1 2 8}, \mathbf{1})}+\operatorname{tr}_{(\mathbf{1}, \mathbf{1 2 8})}-\operatorname{tr}_{(\mathbf{1 6}, \mathbf{1 6})}\right]\left(F_{2}^{p}\right), \quad \operatorname{Tr}_{\text {SUSY }} F_{2}^{2}=0
$$

keeping track of the relative chiralities of the fermions. The latter equation follows for $\mathrm{SO}$ (16) from $\operatorname{tr}_{128} F_{2}^{2}=16 \mathrm{TR} F^{2}$. Moreover, since $\mathrm{SO}(16) \times \mathrm{SO}(16)$ is a subgroup of both $\mathrm{E}_{8} \times \mathrm{E}_{8}$ and $\mathrm{SO}(32)$, we can compare traces in both supersymmetric theories with the non-supersymmetric one, provided that we restrict $F_{2}$ to the adjoint of $\mathrm{SO}(16) \times \mathrm{SO}(16)$. By considering the branching rules of the adjoints of $\mathrm{E}_{8} \times \mathrm{E}_{8}$ and $\mathrm{SO}(32)$ into irreducible representations of $\mathrm{SO}(16) \times \mathrm{SO}(16)$, we find that

$$
\operatorname{Tr}_{\text {SUSY }} F_{2}^{p}=\operatorname{Tr}_{\mathrm{E}_{8} \times \mathrm{E}_{8}} F_{2}^{p}-\operatorname{Tr}_{\mathrm{SO}(32)} F_{2}^{p}
$$

Anomaly cancellation requires the so-called Green-Schwarz mechanism [71, 72] which involves the term

$$
S_{\mathrm{GS}}=\frac{1}{24(2 \pi)^{5} \alpha^{\prime}} \int B_{2} X_{8}
$$

\footnotetext{
${ }^{1}$ We follow a convention where we call the lowest-dimensional irreducible representation of a Lie algebra the "fundamental" representation, even for groups other than $\mathrm{SU}(N)$. Details concerning our trace conventions can be found in appendix A.
} 
in the normalization established in [57]. The polynomial $X_{8}$ is given by (see e.g. [73])

$$
\begin{aligned}
X_{8}^{\mathrm{E}_{8} \times \mathrm{E}_{8}} & =\frac{1}{24} \operatorname{Tr} F_{2}^{4}-\frac{1}{7200}\left(\operatorname{Tr} F_{2}^{2}\right)^{2}-\frac{1}{240} \operatorname{Tr} F_{2}^{2} \mathrm{TR} R_{2}^{2}+\frac{1}{8} \mathrm{TR} R_{2}^{4}+\frac{1}{32}\left(\mathrm{TR} R_{2}^{2}\right)^{2}, \\
X_{8}^{\mathrm{SO}(32)} & =-\frac{1}{24} \operatorname{Tr} F_{2}^{4}+\frac{1}{7200}\left(\operatorname{Tr} F_{2}^{2}\right)^{2}+\frac{1}{240} \operatorname{Tr} F_{2}^{2} \mathrm{TR} R_{2}^{2}-\frac{1}{8} \mathrm{TR} R_{2}^{4}-\frac{1}{32}\left(\mathrm{TR} R_{2}^{2}\right)^{2}, \\
X_{8}^{\mathrm{SUSY}} & =\frac{1}{24} \operatorname{Tr} F_{2}^{4},
\end{aligned}
$$

for the supersymmetric $\mathrm{E}_{8} \times \mathrm{E}_{8}, \mathrm{SO}(32)$, and non-supersymmetric $\mathrm{SO}(16) \times \mathrm{SO}(16)$ theories, respectively. Note that in the non-supersymmetric theory the curvature two-form $R_{2}$ does not appear, hence in this theory the pure (irreducible and reducible) and mixed gravitational anomalies all cancel automatically. Here we have chosen the chiralities of the gravitino, dilatino and gauginos in the $\mathrm{E}_{8} \times \mathrm{E}_{8}$ and $\mathrm{SO}(32)$ precisely opposite to each other; this accounts for the relative sign between the $X_{8}$ 's of both supersymmetric theories. With this convention, one obtains the relation

$$
X_{8}^{\mathrm{SUST}}=X_{8}^{\mathrm{E}_{8} \times \mathrm{E}_{8}}+X_{8}^{\mathrm{SO}(32)}
$$

between the three eight-forms $X_{8}$ for the three heterotic theories. This result arises by making use of the identity (2.4) and that the quadratic trace (2.3) vanishes.

\section{$2.2 \quad$ Heterotic lattices}

The three string theories contain both massless states and states at arbitrary high mass levels, which can be efficiently encoded in lattices. In table 2 we give the full lattices on which the three heterotic theories are constructed. In particular, this displays the root lattice of the gauge group to which their sixteen-component roots $\alpha=\left(\alpha^{I}\right)=\left(\alpha^{1}, \ldots, \alpha^{16}\right)$ belong. The lattices of the $\mathrm{E}_{8} \times \mathrm{E}_{8}$ and $\mathrm{SO}(32)$ theories show that these theories are supersymmetric at every mass level separately, while the $\mathrm{SO}(16) \times \mathrm{SO}(16)$ is not supersymmetric at any mass level.

In ten dimensions the overall notion of positive chirality is of course just a convention. We have chosen the spinorial lattices of $\mathrm{E}_{8} \times \mathrm{E}_{8}$ and $\mathrm{SO}(32)$ such that their chiralities are compatible with the non-supersymmetric twists to the $\mathrm{SO}(16) \times \mathrm{SO}(16)$ theory as we discuss above. In particular, we take the gauginos $\Psi_{+}=\Psi_{\left(\alpha_{0} / 2, \ldots, \alpha_{3} / 2\right)}$ in the $\mathrm{E}_{8} \times \mathrm{E}_{8}$ theory to have positive chirality, i.e. the product of the signs $\alpha_{0} \cdot \ldots \cdot \alpha_{3}=+1$, while the gauginos of the $\mathrm{SO}(32)$ theory are taken to be co-spinors $\Psi_{-}=\Psi_{\left(\alpha_{0} / 2, \ldots, \alpha_{3} / 2\right)}$ with $\alpha_{0} \cdot \ldots \cdot \alpha_{3}=-1$.

\subsection{Non-supersymmetric twists}

The three heterotic theories are closely related on the level of their respective worldsheet theories. For example, the partition function of the $\mathrm{E}_{8} \times \mathrm{E}_{8}$ and $\mathrm{SO}(16) \times \mathrm{SO}(16)$ theories are identical up to some different choices of GSO phases. It is well-known that the $\mathrm{E}_{8} \times \mathrm{E}_{8}$ and $\mathrm{SO}(32)$ theories are T-dual when compactified on a circle with appropriately chosen Wilson lines. Moreover, the $\mathrm{SO}(16) \times \mathrm{SO}(16)$ can be obtained from either the $\mathrm{E}_{8} \times \mathrm{E}_{8}$ or $\mathrm{SO}(32)$ theory by supersymmetry-breaking twists: 


\begin{tabular}{|c||c||c|c|}
\hline \multicolumn{5}{|c|}{ Lattices in heterotic string theories } \\
\hline $\mathrm{N}=1, \mathrm{E}_{8} \times \mathrm{E}_{8}$ & $\mathrm{~N}=1, \mathrm{SO}(32)$ & $\mathrm{N}=0, \mathrm{SO}(16) \times \mathrm{SO}(16)$ & $\supset$ massless states \\
\hline \hline $\mathbf{V}_{4} \otimes \mathbf{R}_{8} \otimes \mathbf{R}_{8}$ & \multirow{2}{*}{$\mathbf{V}_{4} \otimes \mathbf{R}_{16}$} & $\mathbf{V}_{4} \otimes \mathbf{R}_{8} \otimes \mathbf{R}_{8}$ & $(\mathbf{1 , 1 2 0})+(\mathbf{1 2 0}, \mathbf{1})$ \\
$\mathbf{V}_{4} \otimes \mathbf{S}_{8} \otimes \mathbf{S}_{8}$ & & $\mathbf{V}_{4} \otimes \mathbf{S}_{8} \otimes \mathbf{S}_{8}$ & 10D gauge fields \\
\hline $\mathbf{V}_{4} \otimes \mathbf{S}_{8} \otimes \mathbf{R}_{8}$ & \multirow{2}{*}{$\mathbf{V}_{4} \otimes \mathbf{C}_{16}$} & $\mathbf{R}_{4} \otimes \mathbf{C}_{8} \otimes \mathbf{V}_{8}$ & - \\
$\mathbf{V}_{4} \otimes \mathbf{R}_{8} \otimes \mathbf{S}_{8}$ & & $\mathbf{R}_{4} \otimes \mathbf{V}_{8} \otimes \mathbf{C}_{8}$ & - \\
\hline \hline $\mathbf{S}_{4} \otimes \mathbf{R}_{8} \otimes \mathbf{R}_{8}$ & \multirow{2}{*}{$\mathbf{C}_{4} \otimes \mathbf{R}_{16}$} & $\mathbf{S}_{4} \otimes \mathbf{S}_{8} \otimes \mathbf{R}_{8}$ & $(\mathbf{1 , 1 2 8})+(\mathbf{1 2 8}, \mathbf{1})$ \\
$\mathbf{S}_{4} \otimes \mathbf{S}_{8} \otimes \mathbf{S}_{8}$ & & $\mathbf{S}_{4} \otimes \mathbf{R}_{8} \otimes \mathbf{S}_{8}$ & 10D spinors \\
\hline $\mathbf{S}_{4} \otimes \mathbf{S}_{8} \otimes \mathbf{R}_{8}$ & \multirow{2}{*}{$\mathbf{C}_{4} \otimes \mathbf{C}_{16}$} & $\mathbf{C}_{4} \otimes \mathbf{V}_{8} \otimes \mathbf{V}_{8}$ & $(\mathbf{1 6}, \mathbf{1 6})$ \\
$\mathbf{S}_{4} \otimes \mathbf{R}_{8} \otimes \mathbf{S}_{8}$ & & $\mathbf{C}_{4} \otimes \mathbf{C}_{8} \otimes \mathbf{C}_{8}$ & 10D co-spinors \\
\hline
\end{tabular}

Table 2. The different lattices that occur in the eight (or four) different sectors of the supersymmetric $\mathrm{E}_{8} \times \mathrm{E}_{8}, \mathrm{SO}(32)$ and the non-symmetric $\mathrm{SO}(16) \times \mathrm{SO}(16)$ heterotic string theories. V, R, S and $\mathbf{C}$ refer to the vector, root, spinor and co-spinor lattices, respectively. Their subscripts indicate the dimension of these lattices. Consequently, the first lattices in the tensor products classify the states as space-time bosons $(\mathbf{V}, \mathbf{R})$ and fermions $(\mathbf{S}, \mathbf{C})$, respectively, while the remainders correspond to the various gauge representation lattices. We have chosen the chiralities of the spinorial lattices of $\mathrm{E}_{8} \times \mathrm{E}_{8}$ and $\mathrm{SO}(32)$ such that they are compatible with those of the $\mathrm{SO}(16) \times \mathrm{SO}(16)$ theory.

I. A $\mathbb{Z}_{2}$ orbifolding of the $\mathrm{E}_{8} \times \mathrm{E}_{8}$ string with twist $v_{0}=\left(0,1^{3}\right)$ and gauge shift

$$
\begin{aligned}
V_{0}= & \left(1,0^{7}\right)\left(-1,0^{7}\right): \\
& A_{M}^{A} \rightarrow A_{M}^{A}, \quad A_{M}^{X} \rightarrow-A_{M}^{X}, \quad \Psi_{+}^{A} \rightarrow-\Psi_{+}^{A}, \quad \Psi_{+}^{X} \rightarrow \Psi_{+}^{X} .
\end{aligned}
$$

II. A $\mathbb{Z}_{2}$ orbifolding of the $\mathrm{SO}(32)$ string with twist $v_{0}=\left(0,1^{3}\right)$ and gauge shift $V_{0}^{\prime}=\left(1,0^{7}\right)\left(-\frac{1}{2}, \frac{1}{2}^{7}\right)$ :

$$
A_{M}^{A} \rightarrow A_{M}^{A}, \quad A_{M}^{Y} \rightarrow-A_{M}^{Y}, \quad \Psi_{-}^{A} \rightarrow-\Psi_{-}^{A}, \quad \Psi_{-}^{Y} \rightarrow \Psi_{-}^{Y} .
$$

The gauge fields $A_{M}^{A}$ of the $\mathrm{SO}(16) \times \mathrm{SO}(16)$ theory, labeled by $A$, are part of the untwisted sector in either case. The additional fermionic matter states can partially be interpreted as untwisted and partially as twisted states: in the non-supersymmetric orbifold of the $\mathrm{E}_{8} \times \mathrm{E}_{8}$ the $(\mathbf{1 2 8}, \mathbf{1})+(\mathbf{1}, \mathbf{1 2 8})$ spinor states $\Psi_{+}^{X}$, labeled by $X$, are untwisted while $(\mathbf{1 6}, \mathbf{1 6})$ cospinor $\Psi_{-}^{Y}$, labeled by $Y$, are twisted. For the non-supersymmetric orbifold of the $\mathrm{SO}(32)$ this assignment is precisely the other way around. All these relations between the three ten-dimensional heterotic string theories are schematically indicated in figure 1.

The actions of the non-supersymmetric twists can be elegantly represented on the tendimensional vector multiplets using $\mathrm{N}=1$ four-dimensional superspace language [74, 75]. One decomposes the gauge fields as $A_{M}=\left(A_{\mu}, A_{a}, A_{\underline{a}}\right)$ with four-dimensional and complexified six-dimensional indices $\mu=0, \ldots 4, a, \underline{a}=1,2,3$ (suppressing the gauge index for now). Their components are contained in vector superfields $\mathcal{V}$ and chiral superfields $Z_{a}$ such that

$$
\frac{1}{2}\left[\bar{D}_{\dot{\alpha}}, D_{\alpha}\right] \mathcal{V}\left|=\sigma_{\dot{\alpha} \alpha}^{\mu} A_{\mu}, \quad Z_{a}\right|=A_{a}
$$


where | denotes setting all Grassmann variables $\theta$ to zero. The ten-dimensional gaugino components $\Psi_{ \pm}$are then represented as

$$
\begin{aligned}
& \Psi_{\left(\frac{\alpha}{2}, \frac{\alpha}{2}\right)}{ }^{:} W_{\alpha}\left|=\lambda_{\alpha}, \quad \Psi_{\left(\frac{\alpha}{2}, \underline{\frac{\alpha}{2}}^{-\frac{\alpha}{2}}{ }^{2}\right)}: \frac{1}{\sqrt{2}} D_{\alpha} Z_{a}\right|=\psi_{a \alpha}, \\
& \Psi_{\left(\frac{\alpha}{2},-\frac{\alpha}{2}^{3}\right)}: W_{\alpha}\left|=\lambda_{\alpha}, \quad \Psi_{\left(\frac{\alpha}{2},-\frac{\alpha}{2}, \frac{\alpha}{2}^{2}\right)}: \frac{1}{\sqrt{2}} D_{\alpha} Z_{a}\right|=\psi_{a \alpha},
\end{aligned}
$$

for $\mathrm{E}_{8} \times \mathrm{E}_{8}$ and $\mathrm{SO}(32)$, respectively. Here $W_{\alpha}=-\frac{1}{4} \bar{D}^{2}\left(e^{-V} D_{\alpha} e^{V}\right)$ is the super field strength of the four-dimensional superspace and the underline denotes permutation of the entries. The actions (2.8a) and (2.8b) of the non-supersymmetric twists of the $\mathrm{E}_{8} \times \mathrm{E}_{8}$ and $\mathrm{SO}(32)$ theories on these superfields are given by

I. The $\mathbb{Z}_{2}$ twist of the $\mathrm{E}_{8} \times \mathrm{E}_{8}$ gauge multiplets:

$$
\left(\theta^{\alpha}, \bar{\theta}^{\dot{\alpha}}\right) \rightarrow-\left(\theta^{\alpha}, \bar{\theta}^{\dot{\alpha}}\right), \quad \mathcal{V}^{A} \rightarrow \mathcal{V}^{A}, \quad \mathcal{V}^{X} \rightarrow-\mathcal{V}^{X}, \quad Z_{a}^{A} \rightarrow Z_{a}^{A}, \quad Z_{a}^{X} \rightarrow-Z_{a}^{X} .
$$

II. The $\mathbb{Z}_{2}$ twist of the $\mathrm{SO}(32)$ gauge multiplets:

$$
\left(\theta^{\alpha}, \bar{\theta}^{\dot{\alpha}}\right) \rightarrow-\left(\theta^{\alpha}, \bar{\theta}^{\dot{\alpha}}\right), \quad \mathcal{V}^{A} \rightarrow \mathcal{V}^{A}, \quad \mathcal{V}^{Y} \rightarrow-\mathcal{V}^{Y}, \quad Z_{a}^{A} \rightarrow Z_{a}^{A}, \quad Z_{a}^{Y} \rightarrow-Z_{a}^{Y} .
$$

The simultaneous reflection of all Grassmann variables ensures that the $\mathrm{SO}(16) \times \mathrm{SO}(16)$ gaugino components are all projected out by this non-supersymmetric twist. Consequently, if we want to use four-dimensional $\mathrm{N}=1$ superfields to represent the non-supersymmetric $\mathrm{SO}(16) \times \mathrm{SO}(16)$ theory, we have the following lowest non-vanishing components,

$$
\begin{aligned}
& \frac{1}{2}\left[\bar{D}_{\dot{\alpha}}, D_{\alpha}\right] \mathcal{V}^{A}\left|=\sigma_{\dot{\alpha} \alpha}^{\mu} A_{\mu}^{A}, \quad Z_{a}^{A}\right|=A_{a}^{A}, \\
& W_{\alpha}^{X}\left|=\lambda_{\alpha}^{X}, \quad D_{\alpha} Z_{a}^{X}\right|=\psi_{\alpha a}^{X}, \quad W_{\alpha}^{Y}\left|=\lambda_{\alpha}^{Y}, \quad D_{\alpha} Z_{a}^{Y}\right|=\psi_{\alpha a}^{Y},
\end{aligned}
$$

of the superfields, $\mathcal{V}^{A}, Z_{a}^{A}, Z_{a}^{X}$, and $Z_{a}^{Y}$ defined above. In addition, the $\mathrm{SO}(16) \times \mathrm{SO}(16)$ adjoint vector and chiral multiplets, $\mathcal{V}^{A}$ and $Z_{a}^{A}$, may contain non-vanishing auxiliary fields, $D^{A}$ and $F_{a}^{A}$, respectively. Just as in the supersymmetric theories, using their algebraic equations of motion these auxiliary components can be expressed in terms of the dynamical fields in the theory. In other words in the non-supersymmetric theory the superfields define very convenient short-hand notations.

\section{$3 \quad$ Smooth backgrounds}

When one starts from a non-supersymmetric theory, there seems to be no reason to consider backgrounds that would preserve supersymmetry by themselves. However, as was pointed out in [43] it may be very convenient to consider such backgrounds as there are more computational tools available.

We focus primarily on line bundle backgrounds, which only satisfy the Bianchi identities in cohomology. This means that one is not really working on a smooth Calabi-Yau manifold, but rather on a more complicated torsion manifold. The corrections to the BPS equations, the so-called Strominger system [67, 76-82], give the next-to-leading corrections in the $\alpha^{\prime}$-expansion. Given that we only work to leading order in $\alpha^{\prime}$, we will ignore complications due to torsion in the following. 


\subsection{Calabi-Yau manifolds}

A very crude characterization of a Calabi-Yau manifold is given by its Hodge numbers $h_{11}$ and $h_{21}$ which count the number of independent closed (1,1)- and (2,1)-forms or their corresponding hyper-surfaces.

Topological data. In more detail, any Calabi-Yau manifold $X$ contains a set of complex codimension one hypersurfaces called divisors. A large class of so-called toric Calabi-Yau spaces are constructed as hypersurfaces in some toric ambient space. Toric divisors of this ambient space are defined by simply setting one of the homogeneous coordinates to zero, i.e. $\mathcal{D}_{a}:=\left\{z_{a}=0\right\}$. In general these divisors are dependent, which means that there are various linear equivalence relations among them. We denote a basis of $h_{11}$ independent elements constructed out of the divisors $\mathcal{D}_{a}$ by $\left\{D_{i}\right\}$, and a basis of $h_{11}$ curves by $\left\{C_{i}\right\}$. In this work we mostly focus on so-called "favorable" Calabi-Yau spaces for which this basis of divisors descends from the hyperplane classes of the projective ambient space.

In terms of the aforementioned basis of divisors we have the triple intersection numbers and the second Chern classes evaluated on the $D_{i}$,

$$
\kappa_{i j k}=\int \widehat{D}_{i} \widehat{D}_{j} \widehat{D}_{k}, \quad c_{2 i}=\int_{D_{i}} c_{2}=-\int_{D_{i}} \frac{1}{2} \operatorname{tr}\left(\frac{\mathcal{R}}{2 \pi}\right)^{2} .
$$

Here the curvature two-form $\mathcal{R}$ is $\mathrm{SU}(3)$-valued, so that the trace is evaluated in the fundamental of this holonomy group. In the first expression the closed but not exact $(1,1)$-forms associated to the divisors $D_{i}$ are denoted by $\widehat{D}_{i}$; similarly we denote by $\widehat{C}_{i}$ the $(2,2)$-forms associated to the curves $C_{i}$. In fact, we may take them to be harmonic. Moreover, it is in principle always possible to construct an integral basis of curves and divisors, $\left\{C_{i}\right\}$ and $\left\{D_{i}\right\}$, such that

$$
\int_{C_{i}} \widehat{D}_{j}=\int_{D_{i}} \widehat{C}_{j}=\delta_{i j}
$$

Finally, we denote the independent $(2,1)$ two-forms by $\widehat{\omega}_{p}$ with $p=1, \ldots, h_{21}$.

Classical volumes. The Kähler form $J$ can be expanded in the $\widehat{D}_{i}$ basis as

$$
J=a_{i} \widehat{D}_{i},
$$

in terms of the $h_{11}$ Kähler moduli $a_{i}$. The fundamental form $J$ is used to determine the volumes of any curve $C$, divisor $D$ and the manifold $X$ itself:

$$
\operatorname{Vol}(C)=\int_{C} J, \quad \operatorname{Vol}(D)=\frac{1}{2} \int_{D} J^{2}, \quad \operatorname{Vol}(X)=\frac{1}{6} \int_{X} J^{3} .
$$

In the integral basis (3.2) we know that all the moduli satisfy $a_{i}>0$ in the Kähler cone to guarantee that all curves $C_{i}$ are effective, i.e. have positive volume. In fact, we work in the large volume approximation where volumes are much larger than the string scale such that we can reliably neglect higher order $\alpha^{\prime}$-corrections. The volumes of curves, divisors and $X$ read

$$
\operatorname{Vol}\left(C_{i}\right)=a_{i}, \quad \operatorname{Vol}\left(D_{i}\right)=\frac{1}{2} \kappa_{i j k} a_{j} a_{k}, \quad \operatorname{Vol}(X)=\frac{1}{6} \kappa_{i j k} a_{i} a_{j} a_{k}
$$

Consequently, one has $\operatorname{Vol}(X)=\frac{1}{3} \operatorname{Vol}\left(C_{i}\right) \operatorname{Vol}\left(D_{i}\right)$. 
Complete intersection Calabi-Yau manifolds. Manifolds known as Complete Intersection Calabi-Yau (CICY) manifolds are described in terms of intersecting hypersurfaces in projective ambient spaces. All smooth CICYs have been classified in [68] and are available online [69]. Their discrete symmetries have been classified in [70]. Here we focus on the subclass of favorable CICYs, which means that all CICY divisors can be pulled back from the hyperplane divisors of the projective ambient spaces $\otimes_{a} \mathbb{P}^{k_{a}}$. Consequently, a runs from 1 to $h_{11}$. CICYs can be described most easily in terms of their configuration matrix $\Gamma=\left(\Gamma_{a A}\right)$. Each row, labeled by $a$, corresponds to one ambient space $\mathbb{P}^{k_{a}}$ factor and each column, labeled by $A$, corresponds to one polynomial that defines a hypersurface in the ambient space. Thus, an entry $\Gamma_{a A}$ specifies the scaling of the $A^{\text {th }}$ polynomial under the projective scale factor of the $a^{\text {th }}$ projective ambient space factor. Since each polynomial imposes one constraint, we find that $A$ runs from 1 to $\sum_{a} k_{a}-3$ for a CY 3-fold. The first Chern class of a CICY vanishes if

$$
\sum_{A} \Gamma_{a A}=k_{a}+1
$$

In this way, the ambient space follows uniquely from the configuration matrix.

For the calculation of the intersection numbers $\kappa_{i j k}$ and the total Chern class of CICY manifolds we used the methods introduced in [83]:

$$
\begin{aligned}
\kappa_{i j k} & =\prod_{e} \frac{1}{k_{e} !} \frac{\partial^{k_{e}}}{\partial D_{e}^{k_{e}}}\left[\prod_{A}\left(\sum_{a} \Gamma_{a A} D_{a}\right) c(X) D_{i} D_{j} D_{k}\right]_{D=0}, \\
c(X) & =\frac{\prod_{a}\left(1+D_{a}\right)^{k_{a}+1}}{\prod_{B}\left(1+\sum_{b} \Gamma_{b B} D_{b}\right)},
\end{aligned}
$$

where $A, B=1, \ldots, \sum_{a} k_{a}-3$ and $a, b, \ldots=1, \ldots, h_{11}$.

Free quotients. Next, we consider a discrete, freely acting symmetry group $\Gamma$ of finite order $|\Gamma|$ that acts on the coordinates of $X$ as $z \rightarrow g z$. In this work we will assume that $\Gamma$ consists of a single $\mathbb{Z}_{N}$ factor only. Since $\Gamma$ is assumed to act freely, the quotient

$$
\widetilde{X}=X / \Gamma
$$

is again a smooth, but not simply-connected, Calabi-Yau manifold.

We consider cases in which the action of the discrete group $\Gamma$ can be described in terms of the action on the ambient space coordinates. In order to be able to mod out such an action, one has of course to ensure that the Calabi-Yau geometry admits such a symmetry. For example, this poses constraints on the ambient space and the polynomials whose intersections define the CICY. This typically means that some complex structure deformations, counted by $h_{21}$, are frozen. There are essential three ways in which $\Gamma$ can act [70]: 
1. homogeneous coordinates obtain phases,

2. homogeneous coordinates within each $\mathbb{P}^{N}$ factor are permuted,

3. or complete $\mathbb{P}^{N}$ factors are permuted among each other.

In the first case the ambient space divisors $\mathcal{D}_{a}=\left\{z_{a}=0\right\}$ are invariant. The second type of action permutes them among each other, but the corresponding divisor class remains invariant. Hence, if one had chosen it as one of the divisor basis elements $D_{i}$, then it remains inert. In contrast, in the third case one has to form invariant linear combinations of divisors $\left\{D_{i}\right\}$ of $X$. This means that in the third case $h_{11}$ is reduced as well.

\subsection{Line bundles on Calabi-Yaus}

For the gauge background $\mathcal{F}$ we make the simple ansatz that the vector bundle $\mathcal{V}$ is given by a sum of line bundles (see e.g. [61, 62])

$$
\frac{\mathcal{F}}{2 \pi}=\widehat{D}_{i} H_{i}, \quad H_{i}=V_{i}^{I} H_{I},
$$

which are embedded in the Cartan subalgebra of the ten-dimensional gauge group $\mathcal{G}$. This Abelian gauge background is invariant under unbroken gauge transformation. It is characterized by a set of bundle vectors $V_{i}=\left(V_{i}^{I}\right)$, one for each divisor $(1,1)$-form $\widehat{D}_{i}$. The Cartan generators, $H_{I}, I=1, \ldots, 16$, are assumed to be normalized such that ${ }^{2}$

$$
\operatorname{tr}\left(H_{I} H_{J}\right)=\delta_{I J}
$$

Consequently, $\operatorname{tr}\left(H_{i} H_{j}\right)=V_{i} \cdot V_{j}$, where $\cdot$ is the standard (euclidean) inner product of two vectors with sixteen components. The unbroken subgroup $\mathcal{H}$ of the ten-dimensional gauge group $\mathcal{G}$ is generated by this Cartan subalgebra augmented with the creation and annihilation operators associated with the roots $\alpha$ that are perpendicular to all line bundle vectors, $V_{i} \cdot \alpha=0$, for all $i=1, \ldots, h_{11}$. For the $\mathrm{E}_{8} \times \mathrm{E}_{8}$ and $\mathrm{SO}(16) \times \mathrm{SO}(16)$ theories we decompose these bundle vectors w.r.t. observable and hidden gauge group factors as $V_{i}=\left(V_{i}^{\prime}, V_{i}^{\prime \prime}\right)$ and similarly for other quantities where applicable.

Any line bundle background is subject to a number of consistency conditions, which we list in the following:

Flux quantization. The bundle vectors are subject to flux quantization conditions, which ensure that

$$
\int_{C} \frac{\mathcal{F}}{2 \pi}
$$

evaluated on any state $|p\rangle$ in the full string spectrum, is integral for all curves $C$.

If $\left\{D_{k}\right\}$ is an integral basis of divisors satisfying (3.2), this amounts to requiring that all line bundle vectors lie on the lattices

$$
\Lambda_{\mathrm{E}_{8} \times \mathrm{E}_{8}}=\left(\mathbf{R}_{8} \oplus \mathbf{S}_{8}\right) \otimes\left(\mathbf{R}_{8} \oplus \mathbf{S}_{8}\right), \quad \Lambda_{\mathrm{SO}(32)}=\mathbf{R}_{16} \oplus \mathbf{S}_{16},
$$

\footnotetext{
${ }^{2}$ The trace itself is normalized as the trace over the fundamental representation of SU-groups.
} 
in the cases of the $\mathrm{E}_{8} \times \mathrm{E}_{8}$ or $\mathrm{SO}(32)$ heterotic string, respectively. The flux quantization in the non-supersymmetric $\mathrm{SO}(16) \times \mathrm{SO}(16)$ theory requires the bundle vectors to lie on the lattice

$$
\Lambda_{\text {SUSY }}=\mathbf{R}_{8} \otimes \mathbf{R}_{8},
$$

which is contained in both $\mathrm{E}_{8} \times \mathrm{E}_{8}$ and $\mathrm{SO}(32)$ lattices. Consequently, any allowed set of bundle vectors of the $\mathrm{SO}(16) \times \mathrm{SO}(16)$ theory also represents an admissible set for either of the supersymmetric heterotic theories.

Bianchi identities. The Bianchi identities for the $B$-field constitute further consistency conditions on the line bundle gauge background:

$$
\operatorname{tr}\left(\frac{\mathcal{F}}{2 \pi}\right)^{2}-\operatorname{tr}\left(\frac{\mathcal{R}}{2 \pi}\right)^{2}=N_{i} \widehat{C}_{i}
$$

in cohomology, i.e. when integrated over any divisor $D$ of $X$. In the integral basis (3.2) we can interpret $N_{i}$ as the five-brane charge associated to the five-brane wrapping the curve $C_{i}$ :

$$
N_{i}=N_{i}^{\prime}+N_{i}^{\prime \prime}, \quad N_{i}^{\prime}=\kappa_{i j k} V_{j}^{\prime} \cdot V_{k}^{\prime}+c_{2 i}, \quad N_{i}^{\prime \prime}=\kappa_{i j k} V_{j}^{\prime \prime} \cdot V_{k}^{\prime \prime}+c_{2 i},
$$

for the $\mathrm{E}_{8} \times \mathrm{E}_{8}$ or $\mathrm{SO}(16) \times \mathrm{SO}(16)$ theories and

$$
N_{i}=\kappa_{i j k} V_{j} \cdot V_{k}+2 c_{2 i},
$$

for the $\mathrm{SO}(32)$ theory, respectively. When all $N_{i} \geq 0$ the configuration of five-branes preserves the same four-dimensional supersymmetry as the perturbative sector of the $\mathrm{E}_{8} \times \mathrm{E}_{8}$ or $\mathrm{SO}(32)$ theory. For the $\mathrm{E}_{8} \times \mathrm{E}_{8}$ theory, the non-perturbative NS5-brane spectrum involves only a number of tensor multiplets, and hence does not modify the spectrum charged under the perturbative unbroken gauge group $\mathcal{H}$. In contrast, for the $\mathrm{SO}(32)$ theory there are additional matter multiplets in bi-fundamental representations of the unbroken subgroup $\mathcal{H}$ and the non-perturbative groups $\operatorname{Sp}(2 \widetilde{N})$. To the best of our knowledge, it is unknown which additional non-perturbative charged states need to be added to the $\mathrm{SO}(16) \times \mathrm{SO}(16)$ theory; we will present a suggestion for this in section 8 .

Donaldson-Uhlenbeck-Yau equations. An additional requirement on the line bundle is that the DUY equations,

$$
\frac{1}{2} \int J^{2} \frac{\mathcal{F}}{2 \pi}=\operatorname{Vol}\left(D_{i}\right) V_{i}^{I}=0
$$

can be satisfied. At first this seems to impose a condition on the moduli only, encoded in the volumes of the divisors $D_{i}$, but in fact it leads to stringent restrictions on the possible line bundle vectors. This comes about because one has to ensure that the zero-vector can be obtained from a linear combination of the $V_{i}^{I}$ with positive coefficients only.

We have not included the one-loop correction terms $[55,84]$ for the following reasons: first of all it is often possible to absorb this one-loop correction by appropriately shifting the volumes of the divisors. Only when one has an embedding in both $\mathrm{E}_{8}$ gauge group 
factors, it is not generically possible to do so. Second, the form of this correction is not known for the compactification of the non-supersymmetric $\mathrm{SO}(16) \times \mathrm{SO}(16)$ theory. More importantly, we expect other effects to be generated at one-loop order (e.g. appearance of tachyons) in the non-supersymmetric theory. Hence, for that reason also, our analysis will focus on the weak coupling limit of the theory, where such corrections can be neglected. Note that the DUY equations are homogeneous at tree level, such that at this order one can always go to a large volume point in moduli space.

Equivariant line bundles. By accompanying the action of the freely acting symmetry $\Gamma$, which was used to obtain the non-simply-connected Calabi-Yau $\widetilde{X}=X / \Gamma$ from the Calabi-Yau $X$, with an action on the gauge degrees of freedom,

$$
A(z) \rightarrow A(g z)=W_{g} A(z) W_{g}^{-1},
$$

we can induce a further gauge symmetry breaking: $\mathcal{H} \rightarrow \widetilde{\mathcal{H}}$. In practice, such a freely acting Wilson line $W_{g}$ induces a non-local gauge symmetry breaking, typically chosen such that a GUT subgroup of $\mathcal{H}$ in the upstairs description is broken down to the SM group in the downstairs picture. However, before we divide out a freely acting symmetry in a heterotic theory, we need to make sure that the bundle is equivariant under (i.e. compatible with) the action:

$$
\mathcal{F}(z) \rightarrow \mathcal{F}(g z)=W_{g} \mathcal{F}(z) W_{g}^{-1},
$$

for all $g \in \Gamma$. We assume that the generator of the freely acting symmetry can be diagonalized simultaneously with the line bundle flux, therefore it may be written as $W_{g}=\exp \left(2 \pi i W^{I} H_{I}\right)$.

In the part on free quotients in subsection 3.1 we consider three possible $\Gamma$-actions on the geometry. As each of the basis divisors $D_{k}$ is invariant under the first two actions listed there, the line bundles constructed in the upstairs picture are automatically invariant. For the third type of freely acting symmetry, which permutes various $\mathbb{P}^{N}$ factors, a simple way to ensure equivariance is to require that the corresponding gauge vectors are identical. As this reduces the number of independent line bundle vectors, this tightens the constraints on having large volume solutions to the DUY equations and satisfying the Bianchi identities without NS5-branes.

\section{Spectra of smooth Calabi-Yau compactifications}

In this section we discuss methods to compute the massless spectrum of smooth compactifications of the two supersymmetric heterotic string theories and the non-supersymmetric $\mathrm{SO}(16) \times \mathrm{SO}(16)$ theory.

\subsection{Massless charged chiral 4D spectrum}

For many applications it is sufficient to compute only the charged chiral spectrum. To this end it is convenient to use the multiplicity operator $\mathcal{N}(X)$, see e.g. $[61,63,85,86]$, 
that counts the number of chiral states. It was obtained in [61] by integrating the tendimensional anomaly polynomial $I_{12 \mid \mathbf{R}}$ over the internal Calabi-Yau manifold:

$$
I_{6 \mid \mathbf{R}}(X)=\int_{X} I_{12 \mid \mathbf{R}}=\frac{1}{2(2 \pi)^{2}} \operatorname{tr}_{\mathbf{R}}\left[\mathcal{N}(X)\left(\frac{1}{6} F_{2}^{3}-\frac{1}{48}\left(\mathrm{TR} R_{2}^{2}\right) F_{2}\right)\right] .
$$

Here $\mathbf{R}$ is the representation which the ten-dimensional states are transforming in, $R_{2}$ is the four-dimensional curvature two-form and $F_{2}$ is the gauge field strength of the unbroken gauge group $\mathcal{H}$ in four dimensions. Consequently, the multiplicity operator,

$$
\mathcal{N}(X)=\frac{1}{(2 \pi)^{3}} \int_{X}\left\{\frac{1}{6} \mathcal{F}_{2}^{3}-\frac{1}{24} \operatorname{tr}\left(\mathcal{R}_{2}^{2}\right) \mathcal{F}_{2}\right\}=\frac{1}{6} \kappa_{i j k} H_{i} H_{j} H_{k}+\frac{1}{12} c_{2 i} H_{i},
$$

can be evaluated on each of the weights $p$ of the appropriate representations given in table 1 using that $H_{i}(p)=V_{i} \cdot p$.

The multiplicity operator was obtained in the context of the supersymmetric heterotic $\mathrm{E}_{8} \times \mathrm{E}_{8}$ string (and of course applies to the $\mathrm{SO}(32)$ case in a straightforward way). In ref. [43] it was argued that this formula can also be employed for the non-supersymmetric $\mathrm{SO}(16) \times \mathrm{SO}(16)$ theory: to determine both the chiral fermionic and bosonic spectra one has to suitably choose the representations $\mathbf{R}$ and keep track of the ten-dimensional chirality. To compute the number of complex scalars we take for $\mathbf{R}$ the adjoint representation of $\mathrm{SO}(16) \times \mathrm{SO}(16)$, while for chiral fermions in four dimensions we take states in the spinor representation of either of the two $\mathrm{SO}(16) \mathrm{s}$, or states in the bi-fundamental $(\mathbf{1 6}, \mathbf{1 6})$. Because of the opposite ten-dimensional chirality, the latter states transform in charge conjugate representations as compared to the states resulting from the spinor representations.

The multiplicity operator can also be evaluated on the free quotient $\widetilde{X}=X / \Gamma$ : the downstairs multiplicity of any chiral state in the upstairs spectrum is simply given by

$$
\mathcal{N}(\widetilde{X})=\mathcal{N}(X / \Gamma)=\frac{1}{|\Gamma|} \mathcal{N}(X)
$$

This can be seen as follows: by writing $X=\bigcup_{g} \widetilde{X}^{g}$, where $\widetilde{X}^{g}$ denotes the image of $\widetilde{X} \supset X$ under $g \in \Gamma$, we can decompose the integral in (4.1) in $|\Gamma|$ pieces. Using the fact that $\Gamma$ acts freely on the geometry and equivariantly on the bundle, we see that this gives $|\Gamma|$ equal contributions.

\subsection{Dirac and Hirzebruch-Riemann-Roch indices}

To give additional motivation for using this formula to compute the spectra for both fermions and bosons, we resort to the following index theorems:

- The net spectrum of chiral fermions is determined by the Dirac index [87]

$$
\operatorname{ind}_{\operatorname{Dirac}}(X, \mathcal{V})=\int_{X} \operatorname{ch}(\mathcal{V}) \widehat{\mathrm{A}}(X),
$$

where ch is the Chern character of the bundle and $\widehat{A}$ the roof-genus. 
- Similarly, the net spectrum of complex bosons is characterized by the HirzebruchRiemann-Roch index theorem [65, 87, 88]

$$
\operatorname{ind}_{\mathrm{HRR}}(X, \mathcal{V})=\int_{X} \operatorname{ch}(\mathcal{V}) \operatorname{Td}(X)
$$

which involves the Todd class Td instead of the $\widehat{A}$ class.

Using the splitting principle, a vector bundle $\mathcal{V}$ can be represented as $\mathcal{V}=\oplus_{j} \mathcal{L}_{j}$ where $\mathcal{L}_{j}$ are line bundles. Since these are one-dimensional, they are characterized completely in terms of their first Chern class. Letting $x_{j}=c_{1}\left(\mathcal{L}_{j}\right)$, we can express the Chern class, the Chern character, the Todd class, and the $\widehat{\mathrm{A}}$ class as the products

$$
\begin{aligned}
\mathrm{c}(\mathcal{V}) & =\prod_{j}\left(1+x_{j}\right), & \operatorname{ch}(\mathcal{V}) & =\sum_{j} e^{x_{j}} \\
\operatorname{Td}(\mathcal{V}) & =\prod_{j} \frac{x_{j}}{1-e^{-x_{j}}}, & \widehat{A}(\mathcal{V}) & =\prod_{j} \frac{x_{j} / 2}{\sinh \left(x_{j} / 2\right)},
\end{aligned}
$$

respectively. Expanding the Todd and $\widehat{\mathrm{A}}$ classes to third order in terms of the Chern classes,

$$
\begin{aligned}
\operatorname{Td}(X) & =1+\frac{1}{2} \mathrm{c}_{1}(X)+\frac{1}{12}\left(\mathrm{c}_{1}^{2}(X)+\mathrm{c}_{2}(X)\right)+\frac{1}{24} \mathrm{c}_{1}(X) \mathrm{c}_{2}(X), \\
\widehat{A}(X) & =1-\frac{1}{24} \mathrm{c}_{1}^{2}(X)+\frac{1}{12} \mathrm{c}_{2}(X),
\end{aligned}
$$

with $\mathrm{c}_{1}(X)=\sum_{j} x_{j}, \mathrm{c}_{2}(X)=\sum_{i>j} x_{i} x_{j}$, shows that the indices (4.4) and (4.5) agree when the compactification manifold has vanishing first Chern class $c_{1}(X)=0$. Furthermore, these indices reproduce the multiplicities determined by the multiplicity operator (4.2) evaluated on the appropriate weights.

\subsection{Beyond the chiral spectrum}

While the multiplicity operator (4.2) gives us the net chiral multiplicity of the charged states, determining the number of truly vector-like pairs is more difficult. In general one would have to compute individual dimensions of cohomology groups of appropriate wedge products of the line bundles, rather than just their alternating sum which appears in the indices. Determining the full spectrum provides a strong cross-check on the chiral spectrum determined by the multiplicity operator. Moreover, knowing the full spectrum is important in order to be able to check whether there are exotics in the spectrum that are vector-like with respect to all line bundle charges. For example, if we want to investigate whether we have the exact fermionic spectrum of the MSSM without exotics, we need to show that we have exactly $3|\Gamma|$ 10-plets and no $\overline{\mathbf{1 0}}$-plets on the SU(5) GUT level, where $|\Gamma|$ is the order of the discrete Wilson line.

In order to compute the full spectrum via cohomology group dimensions we make use of the Mathematica package cohomcalg $[89,90]$. The idea behind this code is the following: the spectrum of a line bundle background on a CICY can be determined by computing 
the ambient space vector bundle cohomology and subsequently restricting it to the CalabiYau via the so-called Koszul sequence. This is an exact sequence for a hypersurface in codimension $r$ twisted by the bundle $\mathcal{V}$

$$
\left.0 \rightarrow \mathcal{V} \otimes \bigwedge^{r} N^{*} \rightarrow \mathcal{V} \otimes \bigwedge^{r-1} N^{*} \rightarrow \ldots \rightarrow \mathcal{V} \otimes \bigwedge^{1} N^{*} \rightarrow \mathcal{V} \rightarrow \mathcal{V}\right|_{X} \rightarrow 0
$$

where $N^{*}$ is the dual normal bundle of the CICY, i.e. of the intersection locus of the hypersurface equations. We are interested in the last part $\left.\mathcal{V}\right|_{X}$. By introducing auxiliary sheaves we can break this exact sequence into several short exact sequences. These give rise to long exact sequences in cohomology. We can compute the dimension of these cohomologies in the ambient space. Due to the exactness of (4.8), this allows us to determine the cohomology of the $\left.\mathcal{V}\right|_{X}$ part we are interested in but which we cannot compute directly. Exactness implies that the alternating sum of the dimension of the cohomology groups add up to zero. Thus in cases where "enough" ambient space cohomology groups are trivial, i.e. when no more than three consecutive positions in the Koszul are non-zero, the dimensions of the cohomology groups follow uniquely. In cases where more consecutive positions are non-vanishing, all we know is that their alternating sum equals zero. Due to this, the cohomcalg-package does not determine the dimensions of the cohomology groups uniquely in such cases. In order to resolve the ambiguity one has to construct explicitly the maps between the cohomology groups and work out their kernels and images.

Higgs doublet pairs in supersymmetric $4 \mathrm{D}$ effective theories. The determination of the full spectrum is in particular relevant to determine the Higgs sector. Let us first consider Calabi-Yau compactifications of one of the supersymmetric heterotic strings. In addition to having at least the $3|\Gamma| \overline{\mathbf{5}}$-plets, which contain the left-handed SM quarks and leptons, we need at least one pair of $\mathbf{5}-\overline{\mathbf{5}}$-plets which contain a SM Higgs candidate. (As is well-known in the MSSM one needs a pair of Higgs doublets in order to cancel anomalies induced by the Higgsinos.) Note that such Higgs candidate pairs of $\mathbf{5}-\overline{\mathbf{5}}$-plets need to behave very differently under the freely acting symmetry $\Gamma$ than the $\overline{\mathbf{5}}$ that contain the left-handed quarks and leptons: from the latter we want to retain the full $\overline{\mathbf{5}}$-plets since their triplets correspond to the down-type quarks; merely their multiplicity should be reduce by $|\Gamma|$. In contrast, $\mathbf{5}-\overline{\mathbf{5}}$-plet pairs have to become split multiplets, such that the Higgs doublets survive while the triplets are projected out by the Wilson line. This means that for the remaining vector-like Higgs pair the overall multiplicity stays zero.

Higgs doublet(s) in non-supersymmetric 4D effective theories. In nonsupersymmetric four-dimensional theories it is no problem to have just a single Higgs, since it is a scalar and thus does not produce any anomalies. However, from our previous discussion we infer that it is impossible to obtain just a single Higgs doublet: as all chiral representations have a multiplicity which is divisible by $|\Gamma|$, we need to start with the lowest possible number, i.e. $|\Gamma|$, of additional 5 -plets that can host the SM Higgs, in order to keep exactly one Higgs doublet in the downstairs spectrum. However, after dividing out the freely acting Wilson line we will then obtain one doublet and one triplet as the surviving $\mathbf{5}$-plet is merely branched. Alternatively, as in the supersymmetric case, we 
could start with a vector-like pair (such that the combined multiplicity is zero) and then divide by the freely acting symmetry such that the triplets are projected out. But then we have a pair of Higgs doublets rather than a single one. Consequently, we either have only one Higgs together with its color triplet partner or we have at least one pair of vector-like Higgs doublets.

Note that the situation is different for orbifold compactifications of the $\mathrm{SO}(16) \times \mathrm{SO}(16)$ theory: indeed, in [43] various orbifold models with a single Higgs doublet were obtained. This statement is not in conflict with our previous observations on smooth manifolds: the orbifold gauge shift and discrete Wilson lines in these orbifold models were constructed such that they break the gauge group directly to the SM group. In other words no freely acting symmetry was needed to break an intermediate GUT group down to the SM, but it is precisely such a freely acting symmetry that lead us to the conclusion above.

\section{$5 \quad$ Effective theories in four dimensions}

For the compactifications of the supersymmetric $\mathrm{E}_{8} \times \mathrm{E}_{8}$ and $\mathrm{SO}(32)$ theories we can use the familiar $\mathrm{N}=1$ superspace formalism involving the Kähler potential, superpotential and the gauge kinetic functions, to fully characterize the resulting effective theories in four dimensions. Moreover, as long as we neglect $\alpha^{\prime}$ and $g_{s}$ corrections we may even use this language to efficiently describe the effective theory of Calabi-Yau compactifications of the non-supersymmetric $\mathrm{SO}(16) \times \mathrm{SO}(16)$ string as well. However, in this case we use these functions as convenient short hands to describe specific bosonic and fermionic terms of the action. When we go beyond the leading order, this formalism breaks down since loops involving bosons and fermions are not identical anymore. However, as long as we consider Green-Schwarz interactions, that are directly related to anomaly cancellation, we can still trustworthily compute the corresponding axion couplings as in supersymmetric theories.

\subsection{Effective four-dimensional $\mathrm{N}=1$ actions for $\mathrm{E}_{8} \times \mathrm{E}_{8}$ and $\mathrm{SO}(32)$ compacti- fications}

Below we give the Kähler potential $\mathcal{K}$, superpotential $\mathcal{W}_{B}$ and gauge kinetic function $f$ which characterize the compactification of the supersymmetric heterotic string theories. We review how these functions can be (partially) inferred from dimensional reductions of various terms in the ten-dimensional action (2.1), cf. [91-94]. We expand the Kalb-Ramond two-form and the gauge fields as

$$
\Phi=\varphi_{0}+\varphi, \quad B_{2}=b_{2}+\ell_{s}^{2} \beta_{i} \widehat{D}_{i}, \quad A_{1}=\mathcal{A}_{1}+a_{1}
$$

where $\ell_{s}^{2}=(2 \pi)^{2} \alpha^{\prime}$ denotes the string length. The four-dimensional dilaton is denoted by $\varphi$ and the constant background value of the ten-dimensional dilaton by $\varphi_{0}$. In addition, $\mathcal{A}_{1}$ defines the gauge background with field strength $\mathcal{F}_{2}$, and $a_{1}$ the four-dimensional gauge field one-forms with field strengths $F_{2}$. The Kalb-Ramond two-form can be expanded in terms of harmonic $(1,1)$-forms dual to the divisors $D_{i}$. The fields $\beta_{i}$, appearing in this expansion, are called model-dependent axions; the model-independent axion $\beta_{0}$ is dual to 
the four-dimensional two-form $b_{2}$ :

$$
e^{-4\left(\varphi+\gamma_{0}\right)} *_{4} d b_{2}=d \beta_{0}, \quad G_{\mu \nu}=e^{2\left(\varphi+\varphi_{0}\right)} g_{\mu \nu},
$$

using the four-dimensional Einstein metric $g_{\mu \nu}$, obtained from a four-dimensional Weyl rescaling, to define the Hodge dual $*_{4}$. The four-dimensional Planck scale can be read off from the factor in front of the Einstein-Hilbert term to be

$$
\frac{M_{P}^{2}}{8 \pi}=\frac{1}{\kappa_{4}^{2}}=\frac{1}{\kappa_{10}^{2}} \int \frac{1}{6} J^{3}, \quad e^{4 \gamma_{0}}=e^{2 \varphi_{0}}=\ell_{s}^{-6} \int \frac{1}{6} J^{3} .
$$

The constants $\gamma_{0}, \varphi_{0}$ are fixed such that the kinetic terms of gauge fields and their couplings to the model-independent axion $\beta_{0}$, obtained from the Green-Schwarz term (2.5) involving $b_{2}$, can be written as

$$
S_{\mathrm{YM}}=\frac{1}{4} \int d^{4} d^{2} \theta \mathrm{TR}\left[S W^{2}\right]+\text { c.c. },
$$

provided that the defining components of the chiral and vector superfields contain

$$
S\left|\supset \frac{1}{2 \pi}\left[e^{-2 \varphi}+i \beta_{0}\right] \quad \frac{1}{2}\left[\bar{D}_{\dot{\alpha}}, D_{\alpha}\right] \mathcal{V}\right| \supset \sigma_{\dot{\alpha} \alpha}^{\mu} a_{\mu} .
$$

This is compatible with the kinetic terms of the dilaton $\varphi$ and the axion $\beta_{0}$ that arise from the Kähler potential given by

$$
\begin{aligned}
\mathcal{K}= & -\kappa_{4}^{-2} \ln \left[S+\bar{S}-\frac{1}{(2 \pi)^{2}} Q^{I} \mathcal{V}^{I}\right]-\kappa_{4}^{-2} \ln \int_{X} \frac{1}{6} \mathcal{J}^{3} \\
& -\kappa_{4}^{-2} \ln \int_{X} \bar{\Omega} \Omega-\mathcal{K}_{\underline{m} m} \bar{Z}_{\underline{m}} e^{2 q \cdot \mathcal{V}} Z_{m} .
\end{aligned}
$$

The introduction of the vector multiplets $\mathcal{V}^{I}$ in the first term is fixed by determining the gauge connection for the model-independent axion that arise from the Green-Schwarz term (2.5) after using the dualization (5.2). The charges, $Q_{I}$, in

$$
Q^{I} F_{2}^{I}=\frac{1}{24} \frac{1}{(2 \pi)^{3}} \int X_{2,6}
$$

depend strongly on the theory under consideration and are evaluated below. In addition, we have introduced the notation,

$$
\mathcal{J}=-\frac{1}{2}\left(T_{i}+\bar{T}_{i}-\frac{1}{(2 \pi)^{2}} Q_{i}^{I} \mathcal{V}^{I}\right) \widehat{D}_{i}, \quad \Omega=U_{p} \widehat{\omega}_{p}
$$

such that $\mathcal{J} \mid=J /\left(2 \pi \ell_{s}^{2}\right)$ gives the Kähler form $J$. The $(2,1)$-forms $\widehat{\omega}_{p}$ were defined below (3.2).

Here we have defined the chiral superfields

$$
T_{i}\left|\supset \frac{1}{2 \pi}\left[-\frac{a_{i}}{\ell_{s}^{2}}+i \beta_{i}\right], \quad U_{p}\right| \supset \frac{1}{2 \pi} u_{p},
$$


that involve the Kähler and complex structure moduli, respectively. The second term in (5.6) is determined by the kinetic terms of the model-dependent axions $\beta_{i}$ using [95]

$$
\int_{X} \widehat{D}_{i} \wedge * \widehat{D}_{j}=\frac{1}{4 \operatorname{Vol}(X)}\left(\int_{X} J^{2} \widehat{D}_{i}\right)\left(\int_{X} J^{2} \widehat{D}_{j}\right)-\int_{X} J \widehat{D}_{i} \widehat{D}_{j}
$$

where $\operatorname{Vol}(X)$ is given in (3.4). The dependence on the four-dimensional dilaton $\varphi$ dropped out via the Weyl rescaling (5.2).

The coupling to the vector multiplets $\mathcal{V}^{I}$ is determined by collecting the terms proportional to $\widehat{D}_{i}$ in the expansion of $H_{3}$ using the line bundle gauge flux (3.9) and (5.1). Using

$$
H_{3} \supset 2 \pi \ell_{s}^{2} \widehat{D}_{i}\left[d \frac{\beta_{i}}{2 \pi}-\frac{1}{(2 \pi)^{2}} V_{i}^{I} a_{1}^{I}\right],
$$

we find

$$
Q_{i}^{I}=V_{i}^{I}
$$

for the charge of $\beta_{i} / 2 \pi$, i.e. for the imaginary part of $T_{i}$. The final term in (5.6) involves the massless chiral superfields, $Z_{m} \mid=z_{m}$, with charge matrix $q$. Even in Calabi-Yau compactifications of supersymmetric string theories the detailed Kähler potential, encoded in $\mathcal{K}_{\underline{m} m}$ in (5.6), is difficult to determine unless one is on special backgrounds such as orbifolds or uses the standard embedding.

By reducing the kinetic terms of the ten-dimensional gauge fields and the cross-terms in the kinetic terms of the Kalb-Ramond field, one can extract the moduli-dependent part of the gauge kinetic function

$$
S_{\mathrm{YM}} \supset \frac{1}{4} \int \operatorname{Im}\left(T_{i}\right)\left\{\mathrm{TR}\left[\Delta f_{i}^{\prime} F_{2}^{\prime 2}+\Delta f_{i}^{\prime \prime} F_{2}^{\prime \prime 2}\right]+\Delta f_{i}^{I J} F_{2}^{I} F_{2}^{J}\right\} .
$$

The gauge kinetic function coefficients $\Delta f_{i}$ are determined by a reduction of the GreenSchwarz term (2.5),

$$
\begin{aligned}
S_{\mathrm{GS}} & \supset \frac{1}{24(2 \pi)^{3}} \int \beta_{i} D_{i} X_{4,4} \\
& =\frac{1}{4} \int \frac{\beta_{i}}{2 \pi}\left\{\operatorname{TR}\left[\Delta f_{i}^{\prime}{F_{2}^{\prime 2}}^{2}+\Delta f_{i}^{\prime \prime} F_{2}^{\prime \prime 2}\right]+\Delta f_{i}^{J K} F_{2}^{J} F_{2}^{K}+\Delta_{i} \mathrm{TR} R^{2}\right\} .
\end{aligned}
$$

These charges and coefficients determine the factorization of the anomaly polynomial in four dimensions,

$$
\begin{aligned}
4 I_{6}=Q^{I} F_{2}^{I}\left\{\mathrm{TR} F_{2}^{2}-\mathrm{TR} R_{2}^{2}\right\}+Q_{i}^{I} F_{2}^{I}\left\{\operatorname{TR}\left[\Delta f_{i}^{\prime}{F_{2}^{\prime 2}}^{2}+\Delta f_{i}^{\prime \prime} F_{2}^{\prime \prime 2}\right]\right. & +\Delta f_{i}^{J K} F_{2}^{J} F_{2}^{K} \\
& \left.+\Delta_{i} \mathrm{TR} R_{2}^{2}\right\}
\end{aligned}
$$

Here $I_{6}$ is the four-dimensional anomaly polynomial computed directly using the multiplicity operator. Given the prefactor $1 / 4$ in (5.14), we have a normalization factor 4 in the anomaly factorization formula.

The explicit expressions for the charges, $Q_{i}^{I}, Q^{I}$, and the coefficients, $\delta_{\mathrm{GS}}, \Delta f_{i}$, are theory-dependent: 


\section{Supersymmetric $\mathrm{E}_{8} \times \mathrm{E}_{8}$ theory:}

$$
\begin{array}{rlrl}
Q_{i}^{I^{\prime}} & =V_{i}^{\prime I}, & Q_{i}^{I^{\prime \prime}} & =V_{i}^{\prime \prime I}, \\
Q^{I^{\prime}} & =\frac{1}{6} V_{i}^{\prime I}\left(N_{i}^{\prime}-\frac{1}{2} N_{i}^{\prime \prime}\right), & Q^{I^{\prime \prime}} & =\frac{1}{6} V_{i}^{\prime \prime I}\left(N_{i}^{\prime \prime}-\frac{1}{2} N_{i}^{\prime}\right), \\
\Delta f_{i}^{\prime} & =\frac{1}{6}\left(N_{i}^{\prime}-\frac{1}{2} N_{i}^{\prime \prime}\right), & \Delta f_{i}^{\prime \prime} & =\frac{1}{6}\left(N_{i}^{\prime \prime}-\frac{1}{2} N_{i}^{\prime}\right), \\
\Delta f_{i}^{J^{\prime}} K^{\prime} & =\frac{2}{3} \kappa_{i j k} V_{j}^{\prime J} V_{k}^{\prime K}, \quad \Delta f_{i}^{J^{\prime \prime} K^{\prime \prime}}=\frac{2}{3} \kappa_{i j k} V_{j}^{\prime \prime J} V_{k}^{\prime \prime K}, \quad \Delta f_{i}^{J^{\prime} K^{\prime \prime}}=-\frac{1}{3} \kappa_{i j k} V_{j}^{\prime J} V_{k}^{\prime \prime K}, \\
\Delta_{i} & =-\frac{1}{24}\left(\kappa_{i j k} V_{j} \cdot V_{k}+c_{2 i}\right) .
\end{array}
$$

Supersymmetric $\mathrm{SO}(32)$ theory:

$$
\begin{aligned}
-Q_{i}^{I} & =V_{i}^{I}, \quad-Q^{I}=\frac{1}{6} \kappa_{i j k} \operatorname{TR}\left(H_{i} H_{j} H_{k} H_{I}\right)+\frac{1}{12} c_{2 i} V_{i}^{I}, \\
-\Delta f_{i} & =\kappa_{i j k} H_{j} H_{k}+\frac{1}{12} c_{2 i}, \\
-\Delta_{i} & =-\frac{1}{24}\left(\kappa_{i j k} V_{j} \cdot V_{k}+c_{2 i}\right) .
\end{aligned}
$$

\subsection{Elements of the effective $\mathrm{SO}(16) \times \mathrm{SO}(16)$ theory in four dimensions}

At leading order in $g_{s}$ and $\alpha^{\prime}$ we may still employ the $\mathrm{N}=1$ superspace formalism to characterized the bosonic and fermionic fields obtained from a Calabi-Yau compactification of the non-supersymmetric $\mathrm{SO}(16) \times \mathrm{SO}(16)$ theory. The superfields now only include the bosonic or fermionic components present in the non-supersymmetric theories. Therefore, we briefly indicate the non-vanishing dynamical components of the relevant superfields. This approach is similar to the spurion superfield formalism to encode soft supersymmetry breaking.

Tree level superfield action for bosonic fields. In detail we define the following superfields to describe bosonic moduli and matter scalar fields:

$$
S\left|=\frac{1}{2 \pi}\left[e^{-2 \varphi}+i \beta_{0}\right], \quad T_{i}\right|=\frac{1}{2 \pi}\left[-\frac{a_{i}}{\ell_{s}^{2}}+i \beta_{i}\right], \quad U_{p}\left|=\frac{1}{2 \pi} u_{p}, \quad Z_{m}\right|=z_{m},
$$

as in e.g. (5.5) and (5.9) with auxiliary field components but without fermionic components. Similarly the vector multiplet $\mathcal{V}$ does not have any fermionic component:

$$
\frac{1}{2}\left[\bar{D}_{\dot{\alpha}}, D_{\alpha}\right] \mathcal{V} \mid=\sigma_{\dot{\alpha} \alpha}^{\mu} a_{\mu},
$$

as in (5.5). The tree-level action for the scalar moduli and matter fields is obtained from the bosonic Kähler potential

$$
\mathcal{K}_{\text {bos }}=-\kappa_{4}^{-2} \ln [S+\bar{S}]-\kappa_{4}^{-2} \ln \int_{X} \frac{1}{6} \mathcal{J}^{3}-\kappa_{4}^{-2} \ln \int_{X} \bar{\Omega} \Omega-\mathcal{K}_{\overline{\mathrm{bos}}}^{\underline{m} m} \bar{Z}_{\underline{m}} e^{2 q \cdot \mathcal{V}} Z_{m},
$$

with

$$
\mathcal{J}=-\frac{1}{2}\left(T_{i}+\bar{T}_{i}\right) \widehat{D}_{i}
$$

The tree-level gauge kinetic action is given by the familiar expression

$$
S_{\mathrm{YM}}=\frac{1}{4} \int d^{4} d^{2} \theta \mathrm{TR}\left[S W^{2}\right]+\text { c.c. . }
$$


Tree level superfield action for the fermionic fields. In addition, the compactification leads to a set of chiral fermions $\psi_{f}$. We also collect them in chiral superfields $Z_{f}$, such that their only non-vanishing component is given by

$$
\frac{1}{\sqrt{2}} D_{\alpha} Z_{f} \mid=\psi_{f \alpha} .
$$

The tree-level kinetic terms of the chiral fermions $\psi_{f}$ can be encoded in the fermionic Kähler potential

$$
K_{\text {ferm }}=\mathcal{K}_{\text {ferm }}^{f f} \bar{Z}_{\underline{f}} e^{2 q \cdot \mathcal{V}} Z_{f} .
$$

As for the bosonic matter fields the form of this Kähler potential is difficult to obtain for general compactifications.

One-loop induced anomalous axion-gauge couplings. We expect that at the oneloop level one encounters corrections that do not respect the relations that rely on supersymmetry. However, the axion couplings that result directly from the Green-Schwarz mechanism in ten dimensions by reduction can still be computed without further difficulties. These couplings are very important as they provide us with detailed anomaly cancellation checks on the fermionic spectra, as in the case of compactifications of the supersymmetric theories.

The coupling of the axions $\beta_{0}$ and $\beta_{i}$ to the four-dimensional gauge fields is determined by the reduction of the various terms in the ten-dimensional Green-Schwarz action. The anomalous gauge transformations of the axions yield

$$
\delta \beta_{0}=Q_{I} \alpha_{I}, \quad \delta \beta_{i}=Q_{i}^{I} \alpha_{I},
$$

where $\alpha_{I}$ are the Abelian gauge parameters, such that $\delta A_{I \mu}=-(2 \pi) \partial_{\mu} \alpha_{I}$. The charges $Q_{I}$ are defined as in (5.7) and (5.12). By evaluating the integrals in the $\mathrm{SO}(16) \times \mathrm{SO}(16)$ case we find

$$
\begin{aligned}
Q_{i}^{I^{\prime}} & =V_{i}^{\prime I}, \quad Q_{i}^{I^{\prime \prime}}=V_{i}^{\prime \prime I} \\
Q^{I^{\prime}} & =\frac{1}{6} \kappa_{i j k}\left[V_{i}^{\prime I}\left(V_{j}^{\prime} \cdot V_{k}^{\prime}-\frac{1}{2} V_{j}^{\prime \prime} \cdot V_{k}^{\prime \prime}\right)-\operatorname{TR}\left(H_{i}^{\prime} H_{j}^{\prime} H_{k}^{\prime} H_{I}^{\prime}\right)\right], \\
Q^{I^{\prime \prime}} & =\frac{1}{6} \kappa_{i j k}\left[V_{i}^{\prime \prime I}\left(V_{j}^{\prime \prime} \cdot V_{k}^{\prime \prime}-\frac{1}{2} V_{j}^{\prime} \cdot V_{k}^{\prime}\right)-\operatorname{TR}\left(H_{i}^{\prime \prime} H_{j}^{\prime \prime} H_{k}^{\prime \prime} H_{I}^{\prime \prime}\right)\right]
\end{aligned}
$$

The anomalous gauge transformations of the axions lead to a mixing of the axions with the longitudinal parts of the gauge fields and thereby result in massive U(1)s. The anomalous couplings of the axions take the form

$$
\begin{aligned}
S_{\text {axions }}^{\mathrm{GS}} \supset \frac{1}{2 \pi} \int \beta_{0}\left\{\operatorname{tr}{F_{2}^{\prime}}^{2}+\operatorname{tr}{F_{2}^{\prime \prime 2}}^{2}\right\}+\beta_{i}\left\{\operatorname{tr}\left[\Delta f_{i}^{\prime}{F_{2}^{\prime 2}}^{2}+\Delta f_{i}^{\prime \prime} F_{2}^{\prime \prime 2}\right]\right. & +\Delta f_{i}^{I J} F_{2}^{I} F_{2}^{J} \\
& \left.+\Delta_{i} \operatorname{TR}\left(R_{2}^{2}\right)\right\} .
\end{aligned}
$$

The coefficients $\Delta f_{i}$ are determined from the reduction of the Green-Schwarz term (2.5),

$$
S_{\mathrm{GS}} \supset \frac{1}{24(2 \pi)^{3}} \int \beta_{i} D_{i} X_{4,4},
$$


and are given by

$$
\begin{aligned}
\Delta f_{i}^{\prime} & =\kappa_{i j k}\left(\frac{1}{6} V_{j}^{\prime} \cdot V_{k}^{\prime}-\frac{1}{12} V_{j}^{\prime \prime} \cdot V_{k}^{\prime \prime}-H_{j}^{\prime} H_{k}^{\prime}\right) \\
\Delta f_{i}^{\prime \prime} & =\kappa_{i j k}\left(\frac{1}{6} V_{j}^{\prime \prime} \cdot V_{k}^{\prime \prime}-\frac{1}{12} V_{j}^{\prime} \cdot V_{k}^{\prime}-H_{j}^{\prime \prime} H_{k}^{\prime \prime}\right) \\
\Delta f_{i}^{J^{\prime} K^{\prime}} & =\frac{2}{3} \kappa_{i j k} V_{j}^{\prime J} V_{k}^{\prime K}, \Delta f_{i}^{J^{\prime \prime} K^{\prime \prime}}=\frac{2}{3} \kappa_{i j k} V_{j}^{\prime \prime J} V_{k}^{\prime \prime K}, \Delta f_{i}^{J^{\prime} K^{\prime \prime}}=-\frac{1}{3} \kappa_{i j k} V_{j}^{J} V_{k}^{\prime \prime K}, \\
\Delta_{i} & =0
\end{aligned}
$$

\section{Example of a smooth SM-like model}

In this section we discuss an example to illustrate that it is possible to construct semirealistic models by compactifying the non-supersymmetric $\mathrm{SO}(16) \times \mathrm{SO}(16)$ theory on smooth Calabi-Yau manifolds. Concretely we consider a line bundle model on the tetraquadric, i.e. on the CICY 7862 geometry. The relevant topological data for this manifold, i.e. the intersection numbers and second Chern classes, are given by

$$
\kappa_{i j k}=2, \quad c_{2 i}=24
$$

for mutually distinct $i, j, k$ between 1 and 4 . On this geometry we can construct a six generation non-supersymmetric SU(5) GUT theory by choosing the four line bundle vectors that define the gauge bundle according to (3.9) as

$$
\begin{aligned}
& V_{1}=(-1,1,2,-1,-1,-1,2,1)(-1,0,-1,0,0,0,0,0) \\
& V_{2}=\left(\begin{array}{ll}
0,-1,-1,0,0,0,0,0)(1,0,0,-1,0,0,-1,1
\end{array}\right) \\
& V_{3}=\left(\begin{array}{lll}
0,1,1,0,0,0,-2,0)(0,0,-1,2,1,0,2,-2
\end{array}\right) \\
& V_{4}=(1,0,-1,1,1,1,0,-1)(-1,0,2,0,-1,0,0,0)
\end{aligned}
$$

As explained below (3.10) these sixteen-component vectors are split into two eightcomponent pieces that define the embedding of the gauge background into the Cartan subalgebra of the first and second $\mathrm{SO}(16)$ factor, respectively. Using the methods outlined in appendix B, they can be converted into the other basis. ${ }^{3}$

The resulting observable and hidden gauge groups are $G_{\mathrm{obs}}=\mathrm{SU}(5)$ and $G_{\mathrm{hid}}=$ $\mathrm{SU}(3) \times \mathrm{SU}(2) \times \mathrm{SU}(2)$, respectively. This model satisfies the tree-level DUY equations deep inside the Kähler cone: indeed, if we take the volumes of the four divisors to be related,

$$
\operatorname{Vol}\left(D_{1}\right)=\frac{1}{2} \operatorname{Vol}\left(D_{2}\right)=\operatorname{Vol}\left(D_{3}\right)=\operatorname{Vol}\left(D_{4}\right)
$$

the tree-level DUY equations are satisfied.

The full spectrum of a non-supersymmetric six generation SU(5) GUT model with this line bundle background on the geometry at hand is given in table 3 . It contains the

\footnotetext{
${ }^{3}$ Note however that the results of appendix B cannot be applied directly since the matching there is performed for the $\mathrm{E}_{8} \times \mathrm{E}_{8}$ theory and the embedding for the visible sector $\mathrm{SU}(5)$ differs from (B.3) by a Weyl reflection.
} 


\begin{tabular}{|c|c|c|}
\hline & Massless chiral fermions & Massless complex bosons \\
\hline \multirow{3}{*}{ 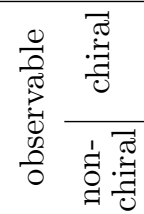 } & $8(\overline{\mathbf{1 0}} ; \mathbf{1}, \mathbf{1}, \mathbf{1})+2(\mathbf{1 0} ; \mathbf{1}, \mathbf{1}, \mathbf{1})$ & \multirow{2}{*}{$16(\mathbf{5} ; \mathbf{1}, \mathbf{1}, \mathbf{1})$} \\
\hline & $+24(\mathbf{5} ; \mathbf{1}, \mathbf{1}, \mathbf{1})+18(\overline{\mathbf{5}} ; \mathbf{1}, \mathbf{1}, \mathbf{1})$ & \\
\hline & - & - \\
\hline \multirow{3}{*}{ 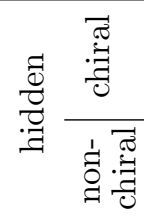 } & $24(\mathbf{1} ; \overline{\mathbf{3}}, \mathbf{1}, \mathbf{1})+20(\mathbf{1} ; \mathbf{3}, \mathbf{1}, \mathbf{1})+2(\mathbf{1} ; \mathbf{3}, \mathbf{2}, \mathbf{1})$ & $16(\mathbf{1} ; \mathbf{3}, \mathbf{1}, \mathbf{1})+12(\mathbf{1} ; \overline{\mathbf{3}}, \mathbf{1}, \mathbf{1})+2(\mathbf{1} ; \overline{\mathbf{3}}, \mathbf{2}, \mathbf{2})$ \\
\hline & $+34(\mathbf{1} ; \mathbf{1}, \mathbf{2}, \mathbf{1})+28(\mathbf{1} ; \mathbf{1}, \mathbf{1}, \mathbf{2})+150(\mathbf{1} ; \mathbf{1}, \mathbf{1}, \mathbf{1})$ & $+4(\mathbf{1} ; \mathbf{1}, \mathbf{2}, \mathbf{2})+80(\mathbf{1} ; \mathbf{1}, \mathbf{1}, \mathbf{1})$ \\
\hline & - & $5(\mathbf{1} ; \mathbf{3}, \mathbf{1}, \mathbf{1})+5(\mathbf{1} ; \overline{\mathbf{3}}, \mathbf{1}, \mathbf{1})$ \\
\hline
\end{tabular}

Table 3. This table gives the full charged spectrum of a six generation non-supersymmetric SU(5) GUT theory on the geometry CICY 7862. The final rows in the observable and hidden sectors displays vector-like states which are not detected by the multiplicity operator. In fact the charged chiral and full spectrum are identical up to the five $\mathbf{3}-\overline{\mathbf{3}}$ pairs in the final row.

chiral spectrum computed using the multiplicity operator evaluated on the various tendimensional states. This model contains vector-like fermionic and bosonic exotics at the chiral level. The last rows in the observable and hidden sectors display the additional non-chiral states that can only be determined by cohomology methods reviewed in subsection 4.3. We see that the number of states that the multiplicity operator misses is very small in this concrete example.

By a freely acting $\mathbb{Z}_{2}$ Wilson line the model becomes a three generation SM-like theory. The Wilson line,

$$
W=\left(\frac{1}{2}, 0,0, \frac{1}{2}, 0,0,0,0\right)(0,0,0,0,0,0,0,0)
$$

breaks the observable gauge group to $G_{\text {obs }}=\mathrm{SU}(2) \times \mathrm{SU}(3) \times \mathrm{U}(1)_{Y}$. Table 4 gives the full spectrum in the downstairs description. Again, the final rows in the observable and hidden sectors give the non-chiral states which the multiplicity operator does not see. We recognize that this model is only SM-like and not a true SM candidate: its spectrum contains eight scalar Higgs doublets, which are all accompanied by scalar color triplets.

\section{Smooth SM-like models from the standard embedding}

As observed in [18] and [43] the standard embedding for the $\mathrm{SO}(16) \times \mathrm{SO}(16)$ string on any Calabi-Yau $X$ yields an SO(10) GUT-like theory. In particular, we have a net number of $h_{21}-h_{11}=\frac{1}{2} \chi(X)$ fermionic 16-plet generations, where $\chi(X)$ is the Euler number of the underlying Calabi-Yau manifold $X$. Via a Wilson line associated to a freely acting symmetry $\Gamma$ there is the possibility to break $\mathrm{SO}(10)$ down to the $\mathrm{SM}$ gauge group with an additional $\mathrm{U}(1)_{\mathrm{B}-\mathrm{L}}$ factor (since a breaking with such discrete Abelian symmetries is always rank-preserving [96]) and reduce the number of chiral generations to three. For this 


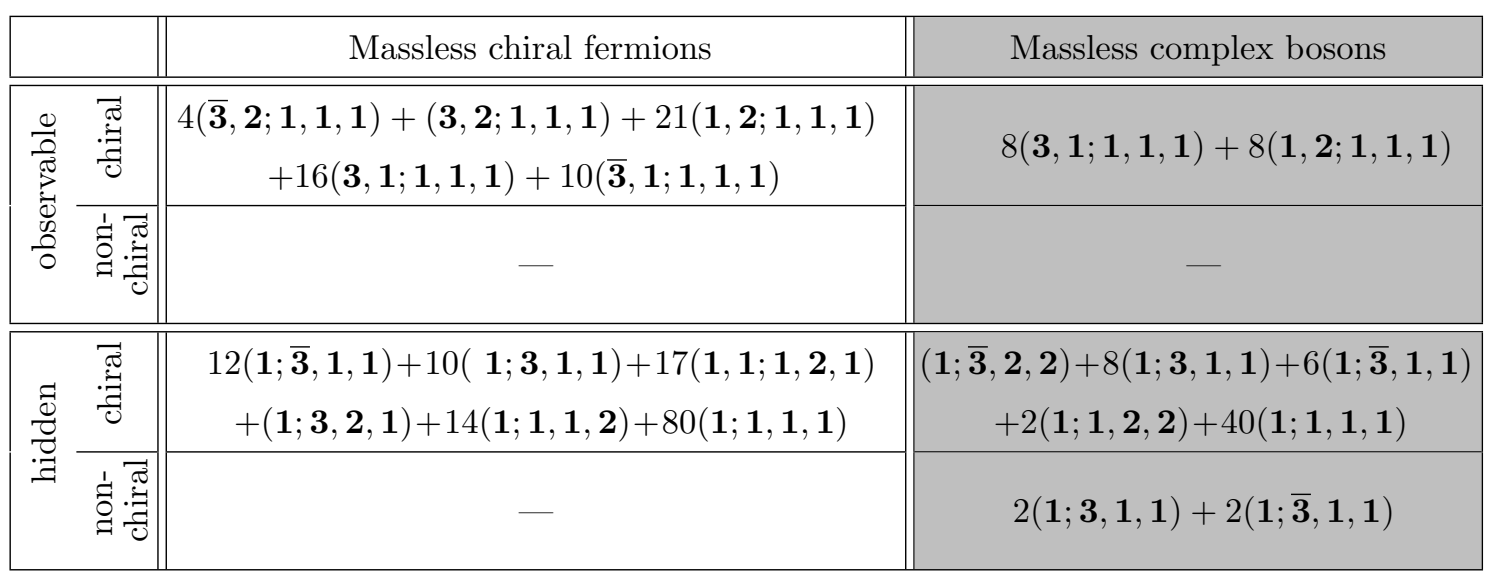

Table 4. This table gives the full charged spectrum of an illustrative non-supersymmetric SM-like model on the geometry CICY 7862. In this model the doublet-triplet splitting problem in the scalar Higgs sector is not resolved.

reason we have to look for a smooth manifold $X$ that satisfies

$$
\frac{1}{2} \chi(X / \Gamma)=\frac{\chi(X)}{2|\Gamma|}=\frac{h_{21}-h_{11}}{|\Gamma|} \stackrel{!}{=} 3
$$

for one of its given freely acting symmetries $\Gamma$.

In order to break $\mathrm{SO}(10)$ down to $G_{\text {obs }}=\mathrm{SU}(3) \times \mathrm{SU}(2) \times \mathrm{U}(1)_{Y} \times \mathrm{U}(1)_{\text {B-L }}$, we need at least a freely acting Abelian $\Gamma=\mathbb{Z}_{N}$ symmetry with $N \geq 4$. To see this, we have depicted the extended Dynkin diagram of the GUT group $\mathrm{SO}(10)$ in figure 3 . Here $-\alpha^{0}=\theta$ denotes the highest root $\theta=a_{i} \alpha^{i}$. The Coxeter labels (or marks) $a_{i}$ for the five simple roots of $\mathrm{SO}(10)$ are given inside the nodes; for $\alpha^{0}$ we define $a_{0}=1$. To determine the unbroken $\mathrm{SO}(10)$ simple roots, when modding out an $\mathbb{Z}_{N}$ Wilson line, we use Dynkin's procedure, as explained in [97] to find the unbroken roots:

$$
N=s_{0}+s_{1}+2\left(s_{2}+s_{3}\right)+s_{4}+s_{5}
$$

where $0 \leq s_{i} \leq N$ for all $i=0, \ldots, 5$. If $s_{i} \neq 0$ the corresponding simple root (and any Weyl reflected root) is broken. Using equation (7.2) we readily compute the lowest order $N$ we need to trigger symmetry breaking down to the gauge group $G_{S M}$ and find the bound $N \geq 4$.

Going through the list of classified CICYs and their freely acting symmetries [68, 70], we find two CICYs with property (7.1): CICY 7246 and CICY 7300. Both geometries have $h_{11}=8, h_{21}=44$ and can support a $\mathbb{Z}_{12}$ Wilson line. If we allow for an additional "hidden" $\mathrm{SU}(2)$ symmetry, i.e. obtain the downstairs gauge group $G_{\text {obs }} \times \mathrm{SU}(2)$, then following (7.2) for the extended Dynkin diagram in figure 3 we can relax the condition for the order of the Wilson line to $N \geq 3$. In the aforementioned list $[68,70]$ there is only one additional geometry satisfying (7.1) with $N=3$ : CICY 536 . 


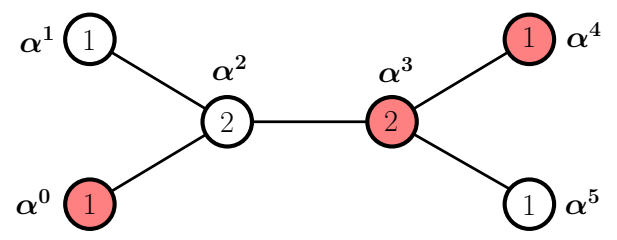

Figure 3. This figure shows the extended Dynkin diagram of $\mathrm{SO}(10)$. For the five simple roots and the extended root the Coxeter labels are given inside the nodes. A breaking to $\mathrm{SU}(3) \times \mathrm{SU}(2)$ is suggested with the colored roots being projected out. All other possible breaking patterns can be obtained by automorphisms of the extended diagram.

\section{Heterotic five-branes in the $\mathrm{SO}(16) \times \mathrm{SO}(16)$ theory}

One may consider the heterotic string in a background of NS5-branes. The properties of NS5-branes strongly depends on the heterotic theory in question: for the $\mathrm{SO}(32)$ and $\mathrm{E}_{8} \times \mathrm{E}_{8}$ their properties have been discussed in the literature, see e.g. [88], while for the $\mathrm{SO}(16) \times \mathrm{SO}(16)$ they are unknown as far as we know.

To establish the properties of NS5-branes within the non-supersymmetric heterotic string, we will make use of the observation that the perturbative spectrum of the theory can be obtained by non-supersymmetric projections of the $\mathrm{SO}(32)$ and $\mathrm{E}_{8} \times \mathrm{E}_{8}$ string. As noted above, the full massless spectrum of the $\mathrm{SO}(16) \times \mathrm{SO}(16)$ involves both untwisted and twisted states, if one starts either from the $\mathrm{SO}(32)$ or $\mathrm{E}_{8} \times \mathrm{E}_{8}$ theory. However, twisted states in one construction are untwisted in the other and vice versa. Hence, by combining the simple orbifold projections within both theories, one has access to the full massless spectrum. Because of this we hope that we can assume the same in the presence of NS5branes: hence we will assume that all NS5-brane states in the $\mathrm{SO}(16) \times \mathrm{SO}(16)$ theory can be understood from the supersymmetry-breaking twist acting on the NS5-branes in the $\mathrm{SO}(32)$ and $\mathrm{E}_{8} \times \mathrm{E}_{8}$ theories. Their anomaly contributions were discussed in detail in [88]; to determine the $\mathrm{SO}(16) \times \mathrm{SO}(16)$ NS5-branes we take inspiration from that discussion. The action of this twist is not completely determined by its action on the perturbative parts of these theories. However, as we will see, anomaly cancellation essentially fixes a unique choice.

Perturbative anomaly contributions. To explain this in detail, we consider a compactification of the $\mathrm{SO}(16) \times \mathrm{SO}(16)$ theory on a smooth K3 with line bundles. The K3 can be characterized by divisors $D_{i}$ with intersection $\kappa_{i j}=D_{i} D_{j}$ and $c_{1}(K 3)=0, c_{2}(K 3)=24$. The perturbative anomaly in six dimensions takes the form

$$
I_{8}^{(\text {full }) \text { pert }}=\int I_{12}^{\text {pert }}=\int-\frac{1}{24}\left(X_{4,0} X_{4,4}+X_{2,2} X_{6,2}+X_{0,4} X_{8,0}\right),
$$

where the reductions of $X_{4}$ and $X_{8}$ read

$$
\begin{aligned}
& X_{4,0}=\mathrm{TR}{F_{2}^{\prime 2}}^{2}+\mathrm{TR} F_{2}^{\prime \prime 2}-\operatorname{TR} R_{2}^{2}, \quad X_{2,2}=2 \mathrm{TR} F_{2}^{\prime} \mathcal{F}_{2}^{\prime}+2 \mathrm{TR} F_{2}^{\prime \prime} \mathcal{F}_{2}^{\prime \prime}, \\
& X_{0,4}=2\left(\operatorname{tr} \mathcal{F}_{2}^{\prime 2}+\operatorname{tr} \mathcal{F}_{2}^{\prime \prime 2}-\operatorname{tr} \mathcal{R}_{2}^{2}\right)
\end{aligned}
$$


and

$$
\begin{aligned}
& X_{4,4}=-6 \mathrm{TR}{F_{2}^{\prime}}^{2} \mathcal{F}_{2}^{\prime 2}-6 \mathrm{TR}{F_{2}^{\prime \prime 2}}^{2} \mathcal{F}_{2}^{\prime \prime 2}+\mathrm{TR}{F_{2}^{\prime 2}}^{2} \operatorname{tr} \mathcal{F}_{2}^{\prime 2}+\mathrm{TR}{F_{2}^{\prime \prime}}^{2} \operatorname{tr} \mathcal{F}_{2}^{\prime \prime 2} \\
& +4\left(\operatorname{tr} F_{2}^{\prime} \mathcal{F}_{2}^{\prime}\right)^{2}+4\left(\operatorname{tr} F_{2}^{\prime \prime} \mathcal{F}_{2}^{\prime \prime}\right)^{2}+ \\
& -\frac{1}{2} \mathrm{TR} F_{2}^{\prime 2} \operatorname{tr} \mathcal{F}_{2}^{\prime \prime 2}-\frac{1}{2} \mathrm{TR} F_{2}^{\prime \prime 2} \operatorname{tr} \mathcal{F}_{2}^{\prime 2}-4 \operatorname{tr}\left(F_{2}^{\prime} \mathcal{F}_{2}^{\prime}\right) \operatorname{tr}\left(F_{2}^{\prime \prime} \mathcal{F}_{2}^{\prime \prime}\right), \\
& X_{6,2}=-4 \mathrm{TR} \mathcal{F}_{2}^{\prime} F_{2}^{\prime 3}-4 \mathrm{TR} \mathcal{F}_{2}^{\prime \prime} F_{2}^{\prime \prime 3}+2 \operatorname{tr}\left(F_{2}^{\prime} \mathcal{F}_{2}^{\prime}\right) \mathrm{TR}{F_{2}^{\prime}}^{2}+2 \operatorname{tr}\left(F_{2}^{\prime \prime} \mathcal{F}_{2}^{\prime \prime}\right) \mathrm{TR} F_{2}^{\prime \prime 2}+ \\
& -\operatorname{tr}\left(F_{2}^{\prime} \mathcal{F}_{2}^{\prime}\right) \mathrm{TR} F_{2}^{\prime \prime 2}-\operatorname{tr}\left(F_{2}^{\prime \prime} \mathcal{F}_{2}^{\prime \prime}\right) \mathrm{TR} F_{2}^{\prime 2} \\
& X_{8,0}=-\mathrm{TR} F_{2}^{\prime 4}-\mathrm{TR} F_{2}^{\prime \prime 4}+\frac{1}{4}\left(\mathrm{TR} F_{2}^{\prime 2}\right)^{2}+\frac{1}{4}\left(\mathrm{TR} F_{2}^{\prime \prime 2}\right)^{2}-\frac{1}{4} \mathrm{TR} F_{2}^{\prime 2} \mathrm{TR} F_{2}^{\prime \prime 2} .
\end{aligned}
$$

The first two contributions in (8.1) are automatically cancelled by the reduction of the perturbative Green-Schwarz mechanism (2.5); therefore we will not consider those contributions further here. For the third and final contribution this is not the case, since the integrated Bianchi identity, the integral over $X_{0,4}$, defines the five-brane charge

$$
N=\frac{1}{2} \int X_{0,4}=\int\left(\operatorname{tr} \mathcal{F}_{2}^{\prime 2}+\operatorname{tr} \mathcal{F}_{2}^{\prime \prime 2}-\operatorname{tr} \mathcal{R}_{2}^{2}\right)=\kappa_{i j} V_{i} \cdot V_{j}+48 .
$$

We see that for $N \neq 0$ the perturbative part of the $\mathrm{SO}(16) \times \mathrm{SO}(16)$ suffers from irreducible anomalies of $\mathrm{SO}(16)$ or appropriate subgroups thereof, but not from an irreducible gravitational anomaly:

$$
I_{8}^{\mathrm{pert}}=-\frac{2 N}{24}\left\{-\mathrm{TR}{F_{2}^{\prime 4}}^{4} \mathrm{TR} F_{2}^{\prime \prime 4}+\frac{1}{4}\left(\mathrm{TR} F_{2}^{\prime 2}\right)^{2}+\frac{1}{4}\left(\mathrm{TR} F_{2}^{\prime \prime 2}\right)^{2}-\frac{1}{4} \mathrm{TR} F_{2}^{\prime 2} \mathrm{TR} F_{2}^{\prime \prime 2}\right\} .
$$

(Here we only give the anomaly contributions that are not cancelled by the reduction of the ten-dimensional Green-Schwarz mechanism.) This means that the NS5-branes in the $\mathrm{SO}(16) \times \mathrm{SO}(16)$ theory have to cancel these irreducible gauge anomalies, but their irreducible gravitational and non-perturbative gauge anomalies all have to vanish independently.

SO(32) NS5-brane anomaly contributions. Next, we briefly discuss the spectra on $\widetilde{N}$ coinciding NS5-branes in the $\mathrm{SO}(32)$ theory, cf. table 5 . To figure out how the nonsupersymmetric twist acts on the NS5-brane spectrum, we first recall that the action on the $\mathrm{SO}(32)$ gauge fields can be represented as

$$
A_{M} \rightarrow U_{\mathrm{SO}} A_{M} U_{\mathrm{SO}}^{T}, \quad \text { where } \quad U_{\mathrm{SO}}=\left(\begin{array}{cc}
\mathbb{1}_{16} & 0 \\
0 & -\mathbb{1}_{16}
\end{array}\right)
$$

We extend the non-supersymmetric twist on the SO(32) NS5-brane fields

$$
\vee \rightarrow U_{\mathrm{Sp}} \vee U_{\mathrm{Sp}}^{T}, \quad \mathrm{H} \rightarrow U_{\mathrm{SO}} \mathrm{H} U_{\mathrm{Sp}}^{T}, \quad \mathrm{C} \rightarrow U_{\mathrm{Sp}} \mathrm{C} U_{\mathrm{Sp}}^{T}, \text { with } U_{\mathrm{Sp}}=\left(\begin{array}{cc}
\mathbb{1}_{2 \widetilde{N}^{\prime}} & 0 \\
0 & -\mathbb{1}_{2 \widetilde{N}^{\prime \prime}}
\end{array}\right)
$$

such that $\widetilde{N}^{\prime}+\widetilde{N}^{\prime \prime}=\widetilde{N}$. The choice of the supersymmetry-breaking twist on the NS5-brane states is a priori not unique even up to similarity transformations. In particular, there may 


\begin{tabular}{|c|c|c|}
\hline \multicolumn{3}{|c|}{$\mathrm{SO}(32)$ NS5-branes } \\
\hline $\begin{array}{c}\operatorname{Sp}(2 \widetilde{N}) 6 \mathrm{D} \\
\text { vector multiplet }\end{array}$ & $\begin{array}{l}\text { Bi-fundamental } \\
\text { half-hypermultiplets }\end{array}$ & $\begin{array}{l}\text { Anti-symmetric } \\
\text { hypermultiplets }\end{array}$ \\
\hline $\mathrm{V} \quad\left([2 \widetilde{N}]_{2}^{+}\right)_{+}$ & $\mathrm{H} \quad(32 ; 2 \widetilde{N})_{-}$ & $\mathrm{C} \quad\left([2 \widetilde{N}]_{2}^{-}\right)_{-}$ \\
\hline $\begin{array}{c}\operatorname{Sp}\left(2 \widetilde{N}^{\prime}\right) \times \operatorname{Sp}\left(2 \widetilde{N}^{\prime \prime}\right) \\
\text { gauge fields }\end{array}$ & $\begin{array}{c}\left(16^{\prime} ; 2 \tilde{N}^{\prime \prime}\right)+\left(16^{\prime \prime} ; 2 \widetilde{N}^{\prime}\right) \\
\text { scalars }\end{array}$ & $\begin{array}{l}\left(2 \tilde{N}^{\prime}, 2 \widetilde{N}^{\prime \prime}\right) \\
\text { scalars }\end{array}$ \\
\hline $\begin{array}{c}\left(2 \widetilde{N}^{\prime}, 2 \tilde{N}^{\prime \prime}\right)_{+} \\
\text {gauginos }\end{array}$ & $\begin{array}{c}\frac{1}{2}\left(16^{\prime} ; 2 \widetilde{N}^{\prime}\right)_{-}+\frac{1}{2}\left(16^{\prime \prime} ; 2 \widetilde{N}^{\prime \prime}\right)_{-} \\
\text {half-hyperinos }\end{array}$ & $\begin{array}{c}\left(\left[2 \tilde{N}^{\prime}\right]_{2}^{-}\right)_{-}+\left(\left[2 \tilde{N}^{\prime \prime}\right]_{2}^{-}\right)_{-} \\
\text {hyperinos }\end{array}$ \\
\hline
\end{tabular}

Table 5. The top part of this table gives the matter spectra on $\widetilde{N}$ coinciding NS5-branes in the heterotic $\mathrm{SO}(32)$ theory. The notation $[2 \widetilde{N}]_{2}^{ \pm}$denotes the totally symmetric/anti-symmetric ranktwo tensor of $\operatorname{Sp}(2 \widetilde{N})$. The subscript \pm on the various representations indicates the six-dimensional chirality of the corresponding fermions. (We use the same convention for the perturbative theories in which the chiralities of $\mathrm{E}_{8} \times \mathrm{E}_{8}$ and $\mathrm{SO}(32)$ are taken to be opposite.) The bottom part of the table displays the remaining states after the non-supersymmetric projection has been performed.

be an additional minus sign in the transformation of the anti-symmetric hypermultiplet $C$. However, as we are then not able to cancel the irreducible $\operatorname{Sp}\left(2 \widetilde{N}^{\prime}\right)$ and $\operatorname{Sp}\left(2 \widetilde{N}^{\prime \prime}\right)$ anomalies, we disregard such possibilities.

The bosonic and fermionic states that survive this supersymmetry-breaking twist (8.7) are given in the last two rows of table 5. The fermionic part of the spectrum produces an anomaly

$$
\begin{aligned}
& I_{8}^{\mathrm{SONS} 5}=\frac{\widetilde{N}^{\prime \prime}-\widetilde{N}^{\prime}}{12}\left(\mathrm{TR} \widetilde{F}_{2}^{\prime 4}-\mathrm{TR} \widetilde{F}_{2}^{\prime \prime 4}\right) \\
& -\frac{15\left(\tilde{N}^{\prime}+\widetilde{N}^{\prime \prime}\right)+2\left(\widetilde{N}^{\prime}-\widetilde{N}^{\prime \prime}\right)^{2}}{128}\left(\frac{1}{45} \mathrm{TR} R_{2}^{4}+\frac{1}{36}\left(\mathrm{TR} R_{2}^{2}\right)^{2}\right) \\
& +\frac{1}{96} \mathrm{TR} R_{2}^{2}\left(\widetilde{N}^{\prime} \mathrm{TR} F_{2}^{\prime 2}+\widetilde{N}^{\prime \prime} \mathrm{TR} F_{2}^{\prime \prime 2}+\left(2 \widetilde{N}^{\prime}-2 \widetilde{N}^{\prime \prime}+6\right) \mathrm{TR} \widetilde{F}_{2}^{\prime 2}+\left(2 \widetilde{N}^{\prime \prime}-2 \widetilde{N}^{\prime}+6\right) \mathrm{TR} \widetilde{F}_{2}^{\prime \prime 2}\right) \\
& -\frac{\widetilde{N}^{\prime}}{24} \mathrm{TR}{F_{2}^{\prime}}^{4}-\frac{\widetilde{N}^{\prime \prime}}{24} \mathrm{TR} F_{2}^{\prime \prime 4}-\frac{1}{8}\left(\mathrm{TR} \widetilde{F}_{2}^{\prime 2}\right)^{2}-\frac{1}{8}\left(\mathrm{TR} \widetilde{F}_{2}^{\prime \prime 2}\right)^{2}+\frac{1}{4} \mathrm{TR} \widetilde{F}_{2}^{\prime 2} \mathrm{TR} \widetilde{F}_{2}^{\prime \prime 2} \\
& -\frac{1}{8} \mathrm{TR}{F_{2}^{\prime}}^{2} \mathrm{TR} \widetilde{F}_{2}^{\prime 2}-\frac{1}{8} \mathrm{TR} F_{2}^{\prime \prime 2} \mathrm{TR} \widetilde{F}_{2}^{\prime \prime 2} \text {. }
\end{aligned}
$$

Here $\widetilde{F}_{2}^{\prime}, \widetilde{F}_{2}^{\prime \prime}$ denote the gauge field strengths of the $\operatorname{Sp}\left(2 \widetilde{N}^{\prime}\right), \operatorname{Sp}\left(2 \widetilde{N}^{\prime \prime}\right)$ groups and TR is the trace in the fundamental of Sp-groups. The overall sign of the anomaly contributions is fixed by the following consideration: in our convention the ten-dimensional chirality of the $\mathrm{SO}(32)$ theory is opposite to that of the $\mathrm{E}_{8} \times \mathrm{E}_{8}$ theory. We require that the NS5-branes coming from the $\mathrm{SO}(32)$ theory preserve the supersymmetry realized in the compactification of the $\mathrm{SO}(32)$ theory. 
The anomaly polynomial contains irreducible anomalies of (various subgroups of) $\mathrm{SO}(16)^{\prime} \times \mathrm{SO}(16)^{\prime \prime}, \mathrm{Sp}\left(2 \widetilde{N}^{\prime}\right)$ and $\mathrm{Sp}\left(2 \widetilde{N}^{\prime \prime}\right)$ and irreducible gravitational anomalies. The irreducible $\operatorname{Sp}\left(2 \widetilde{N}^{\prime}\right)$ and $\operatorname{Sp}\left(2 \widetilde{N}^{\prime \prime}\right)$ anomalies drop out and the irreducible anomalies of (subgroups of) $\mathrm{SO}(16)^{\prime} \times \mathrm{SO}(16)^{\prime \prime}$ cancel those due to the last term in (8.1) (inserting (8.3c)) provided that we choose

$$
\widetilde{N}^{\prime}=\widetilde{N}^{\prime \prime}=2 N
$$

For this choice the irreducible gravitational anomaly remains. It is remarkable that the irreducible parts of those $\mathrm{SO}(16) \times \mathrm{SO}(16)$ anomalies in (8.8) which are independent of $\widetilde{N}^{\prime}, \widetilde{N}^{\prime \prime}$ cancel among themselves.

$\mathbf{E}_{8} \times \mathbf{E}_{8}$ NS5-brane anomaly contributions. We see that the non-supersymmetric projection of the $\mathrm{SO}(32)$ NS5-branes leads to an irreducible gravitational anomaly. To cancel this anomaly we can use the $\mathrm{E}_{8} \times \mathrm{E}_{8}$ NS5-branes that support six-dimensional tensor multiplets $\mathrm{T}_{s}$ and hypermultiplets $\mathrm{H}_{s}, s=1, \ldots, \tilde{n}$. The tensor multiplets include antiself-dual tensors. The scalars in the hypermultiplets parameterize the positions of the NS5-branes on the K3 [98].

As for the $\mathrm{SO}(32)$ NS5-branes, we have to decide how the tensor- and hypermultiplet components transform under the supersymmetry-breaking twist. We take:

$$
\mathrm{T}_{s^{\prime}} \rightarrow \mathrm{T}_{s^{\prime}}, \quad \mathrm{T}_{s^{\prime \prime}} \rightarrow-\mathrm{T}_{s^{\prime \prime}}, \quad \mathrm{H}_{s^{\prime \prime}} \rightarrow \mathrm{H}_{s^{\prime \prime}}, \quad \mathrm{H}_{s^{\prime}} \rightarrow-\mathrm{H}_{s^{\prime}}
$$

with $s^{\prime}=1, \ldots, \tilde{n}^{\prime}$ and $s^{\prime \prime}=1, \ldots, \tilde{n}^{\prime \prime}$, such that $\tilde{n}^{\prime}+\tilde{n}^{\prime \prime}=\tilde{n}$. The surviving spectrum is given in the bottom part of table 6 . The resulting gravitational anomaly reads

$$
I_{8}^{\mathrm{E}_{8} \times \mathrm{E}_{8} \mathrm{NS} 5}=\frac{\tilde{n}^{\prime}}{128}\left(\frac{28}{45} \mathrm{TR} R_{2}^{4}-\frac{8}{36}\left(\mathrm{TR} R_{2}^{2}\right)^{2}\right)+\frac{\tilde{n}^{\prime}+\tilde{n}^{\prime \prime}}{128}\left(\frac{1}{45} \mathrm{TR} R_{2}^{4}+\frac{1}{36}\left(\mathrm{TR} R_{2}^{2}\right)^{2}\right) .
$$

The first contribution comes from the surviving anti-self-dual tensor fields and the second from the surviving hyperinos and tensorinos. Consequently, if we take

$$
\tilde{n}^{\prime \prime}=60 N-29 \tilde{n}^{\prime}
$$

we see that all irreducible gravitational anomalies are cancelled.

Factorization. The remaining reducible anomalies read

$$
\begin{aligned}
I_{8}^{\mathrm{red}}= & \frac{2 N}{96}\left[\mathrm{TR} F^{\prime 2} \mathrm{TR} F^{\prime \prime 2}-\left(\mathrm{TR} F^{\prime 2}\right)^{2}-\left(\mathrm{TR} F^{\prime \prime 2}\right)^{2}+\mathrm{TR} R_{2}^{2}\left(\mathrm{TR} F^{\prime 2}+\mathrm{TR} F^{\prime \prime 2}\right)\right]-\frac{\tilde{n}^{\prime}}{128}\left(\operatorname{TR} R_{2}^{2}\right)^{2} \\
& -\frac{1}{8} \operatorname{TR} F^{\prime 2} \operatorname{TR} \widetilde{F}^{\prime 2}-\frac{1}{8} \operatorname{TR} F^{\prime \prime 2} \operatorname{TR} \widetilde{F}^{\prime \prime 2}+\frac{1}{16} \operatorname{TR} R_{2}^{2}\left(\operatorname{TR} \widetilde{F}^{\prime 2}+\operatorname{TR} \widetilde{F}^{\prime \prime 2}\right) \\
& -\frac{1}{8}\left(\operatorname{TR} \widetilde{F}^{\prime 2}\right)^{2}-\frac{1}{8}\left(\operatorname{TR} \widetilde{F}^{\prime \prime 2}\right)^{2}+\frac{1}{4} \mathrm{TR} \widetilde{F}^{\prime 2} \mathrm{TR} \widetilde{F}^{\prime \prime 2} .
\end{aligned}
$$

This expression is symmetric under the simultaneous exchange of $F_{2}^{\prime 2} \leftrightarrow F_{2}^{\prime \prime 2}$ and $\widetilde{F}_{2}^{\prime 2} \leftrightarrow \widetilde{F}_{2}^{\prime \prime 2}$. Consequently, the anomaly canceling diagrams need to have the same symmetry. 


\begin{tabular}{|c|c|}
\hline \multicolumn{2}{|c|}{$\mathrm{E}_{8} \times \mathrm{E}_{8}$ NS5-branes } \\
\hline \hline Tensor multiplets & Hypermultiplets \\
\hline $\mathrm{T}_{s}$ & $\mathrm{H}_{s}, s=1, \ldots, \tilde{n}$ \\
\hline$\tilde{n}^{\prime}$ anti-self-dual tensors & $\tilde{n}^{\prime \prime}$ complex scalars \\
\hline$\tilde{n}^{\prime \prime}{\text { (tensorinos })_{+}}^{\prime}$ & $\tilde{n}^{\prime}$ (hyperinos) $)_{+}$ \\
\hline
\end{tabular}

Table 6. The top part of this table gives the matter spectra on $\tilde{n}$ coinciding NS5-branes in the $\mathrm{E}_{8} \times \mathrm{E}_{8}$ theory. The bottom part of the table displays the remaining states after the nonsupersymmetric projection has been performed.

The field strength of the anti-symmetric tensors $B_{2}^{s}$ are denoted by $H_{3}^{s}=d B_{2}^{s}+\mathrm{CS}_{3}$, such that

$$
d H_{3}^{s}=\frac{\alpha^{\prime}}{8}\left\{a\left(\mathrm{TR}{F_{2}^{\prime}}^{2}-\mathrm{TR} F_{2}^{\prime \prime 2}\right)+\tilde{a}\left(\mathrm{TR} \widetilde{F}_{2}^{\prime 2}-\mathrm{TR}{\widetilde{F}_{2}^{\prime \prime}}^{2}\right)\right\}
$$

Here $a, \tilde{a}$ are constants to be determined below from anomaly factorization. The relevant part of the six-dimensional NS5-brane action including Green-Schwarz-like Chern-Simons interactions can be represented as

$$
\begin{aligned}
S= & \sum_{s=1}^{\tilde{n}^{\prime}} \int\left\{-\frac{\pi}{\ell_{s}^{4}} H_{3}^{s} \wedge * H_{3}^{s}+\frac{c}{24(2 \pi) \ell_{s}^{2}} B_{2}^{s}\left(\mathrm{TR}{F_{2}^{\prime 2}}^{2}-\mathrm{TR}{F_{2}^{\prime \prime 2}}^{2}\right)\right. \\
& \left.+\frac{1}{24(2 \pi) \ell_{s}^{2}} B_{2}\left(b\left(\mathrm{TR}{F_{2}^{\prime}}^{2}+\mathrm{TR}{F_{2}^{\prime \prime 2}}^{2}\right)+\tilde{b}\left(\mathrm{TR}{\widetilde{F}_{2}^{\prime 2}}^{2} \mathrm{TR} \widetilde{F}_{2}^{\prime \prime 2}\right)-b_{R} \mathrm{TR} R_{2}^{2}\right)\right\},
\end{aligned}
$$

where $b, \tilde{b}, b_{R}, c$ are further constants. This leads to the following anomaly contributions:

$$
\begin{aligned}
& I_{8}^{(1)}=\frac{1}{96}\left[\mathrm{TR} F^{\prime 2}+\mathrm{TR} F^{\prime \prime 2}-\mathrm{TR} R^{\prime 2}\right] \\
& {\left[b\left(\mathrm{TR}{F_{2}^{\prime}}^{2}+\mathrm{TR} F_{2}^{\prime \prime 2}\right)+\tilde{b}\left(\mathrm{TR}{\widetilde{F}_{2}^{\prime 2}}^{2} \mathrm{TR} \widetilde{F}_{2}^{\prime \prime 2}\right)-b_{R} \mathrm{TR} R_{2}^{2}\right],} \\
& I_{8}^{(2)}=\frac{\tilde{n}^{\prime}}{128}\left[a\left(\mathrm{TR}{F_{2}^{\prime 2}}^{2}-\mathrm{TR} F_{2}^{\prime \prime 2}\right)+\tilde{a}\left(\mathrm{TR} \widetilde{F}_{2}^{\prime 2}-\mathrm{TR} \widetilde{F}_{2}^{\prime \prime 2}\right)\right]^{2}, \\
& I_{8}^{(3)}=\frac{\tilde{n}^{\prime} c}{192}\left[a\left(\mathrm{TR}{F_{2}^{\prime 2}}^{2}-\mathrm{TR}{F_{2}^{\prime \prime}}^{2}\right)+\tilde{a}\left(\mathrm{TR}{\widetilde{F}_{2}^{\prime 2}}^{2} \mathrm{TR} \widetilde{F}_{2}^{\prime \prime 2}\right)\right]\left[\mathrm{TR}{F_{2}^{\prime 2}}^{2}-\mathrm{TR} F_{2}^{\prime \prime 2}\right] .
\end{aligned}
$$

These contributions respect the same permutation symmetries as we observed in (8.13). The factor $\tilde{n}^{\prime}$ in the second and third contribution arises because there are $\tilde{n}^{\prime}$ tensors $B_{2}^{s}$ which mediate the anomaly cancellation. They cancel exactly when the coefficients are chosen as

$$
\begin{array}{llll}
\tilde{n}^{\prime}=2 N, & b=\frac{1}{2} N, & \tilde{b}=6, & b_{R}=\frac{3}{2} N, \\
a= \pm \frac{1 \mp \sqrt{1-2 N}}{\sqrt{2 N}}, & \tilde{a}=\frac{4}{\sqrt{2 N}}, & c=3 \frac{\sqrt{1-2 N}}{\sqrt{2 N}} .
\end{array}
$$


Note that there are two more solutions obtained from inverting the signs of $(a, \tilde{a}, c)$ simultaneously which is due to the parameterization in (8.16c). All solutions have $\tilde{n}^{\prime}=2 N$, which means that, using (8.9) and (8.12), $\tilde{N}^{\prime}=\widetilde{N}^{\prime \prime}=\tilde{n}^{\prime}=\tilde{n}^{\prime \prime}$, i.e. the number of the NS5-branes from $\mathrm{SO}(32)$ and $\mathrm{E}_{8} \times \mathrm{E}_{8}$ match.

To summarize we find the rather surprising result that (8.17) has only one genuine solution which has

$$
\tilde{N}^{\prime}=\tilde{N}^{\prime \prime}=\tilde{n}^{\prime}=\tilde{n}^{\prime \prime}=1, \quad \text { and } \quad N=1 / 2,
$$

i.e. a single NS5-brane. This result has been obtained under the following assumptions:

i) We can understand all NS5-brane properties by studying the untwisted sector of the supersymmetry-breaking twist of the supersymmetric $\mathrm{E}_{8} \times \mathrm{E}_{8}$ and $\mathrm{SO}(32)$ theory combined.

ii) The $\mathrm{SO}(32)$ and $\mathrm{E}_{8} \times \mathrm{E}_{8}$ NS5-branes preserve the same supersymmetry as present in the perturbative sector of $\mathrm{SO}(32)$ and $\mathrm{E}_{8} \times \mathrm{E}_{8}$ theories, respectively.

iii) We have ignored the possibility of having states that stretch between the $\mathrm{E}_{8} \times \mathrm{E}_{8}$ and $\mathrm{SO}(32)$-type NS5-branes.

iv) We have made a restrictive ansatz (8.15) for the generalized Green-Schwarz couplings on the NS5-branes.

It would be important to provide either further evidence for this result or to find potential problems and/or generalizations of our arguments. Moreover, we wonder whether we should interpret the five-branes in the $\mathrm{SO}(16) \times \mathrm{SO}(16)$ string as one or two types of NS5-branes. Even more important is the question whether these five-branes could become an additional source for tachyons.

\section{Acknowledgments}

We thank Steve Abel, Keith Dienes and Saul Ramos-Sanchez for valuable discussions and correspondence concerning non-supersymmetric model building. In addition, we would like to thank Ralph Blumenhagen and Anamaria Font for useful discussions on five-branes and anomaly cancellation in the $\mathrm{SO}(16) \times \mathrm{SO}(16)$ context. Finally, we are especially indebted to Patrick K.S. Vaudrevange for numerous discussions comparing supersymmetric and nonsupersymmetric model building.

MB acknowledges the support of the Cluster of Excellence 'Precision Physics, Fundamental Interactions and Structure of Matter' (PRISMA) DGF no. EXC 1098. The work of F.R. was supported by the German Science Foundation (DFG) within the Collaborative Research Center (SFB) 676 "Particles, Strings and the Early Universe". O.L. acknowledges the support by the DAAD Scholarship Programme "Vollstipendium für Absolventen von deutschen Auslandsschulen" within the "PASCH-Initiative". 


\section{A Traces}

A representation $\mathbf{R}=\{|p\rangle\}$ is characterized by a set of vectors $|p\rangle$ corresponding to the weights $p \in W_{\mathbf{R}}=\{p\}$. We identify a representation module $\mathbf{R}$ with its weight system $W_{\mathbf{R}}$ for notational convenience and write $W_{\mathbf{R}}=\mathbf{R}$. Some representations and their weights are indicated for SU and SO-groups in tables 7 and 8, respectively. The dimension of a representation $\mathbf{R}$ is denoted by $|\mathbf{R}|$.

The generators $T_{A}$ of a group $G$ are labeled by $A$. We take the same Cartan generators, denoted by $H_{I}$, in all three heterotic theories. Their eigenvalues are the components of the weights,

$$
H_{I}|p\rangle=p_{I}|p\rangle \text {. }
$$

The remaining generators are denoted by $E_{\alpha}$ where $\alpha$ are the roots of the group, i.e. the weights in the adjoint representation.

The trace of an operator $A_{G}$ over a representation $\mathbf{R}$ is defined as

$$
\operatorname{tr}_{\mathbf{R}}\left(A_{G}\right)=\sum_{p \in \mathbf{R}}\left\langle p\left|A_{G}\right| p\right\rangle .
$$

The subscript $G$ indicates that one is performing the trace of an operator which is a function of objects that are functions of algebra elements associated to the group $G$. The character of an operator $A_{G}$ over a representation $\mathbf{R}$ is defined as

$$
\operatorname{ch}_{\mathbf{R}}\left(A_{G}\right)=\operatorname{tr}_{\mathbf{R}}\left(e^{A_{G}}\right) .
$$

Characters are compatible with direct sums and tensor products in the sense that

$$
\operatorname{ch}_{\mathbf{r} \oplus \mathbf{R}}\left(A_{G}\right)=\operatorname{ch}_{\mathbf{r}}(A)+\operatorname{ch}_{\mathbf{R}}\left(A_{G}\right), \quad \operatorname{ch}_{\mathbf{r} \otimes \mathbf{R}}\left(A_{G}\right)=\operatorname{ch}_{\mathbf{r}}\left(A_{G}\right) \operatorname{ch}_{\mathbf{R}}\left(A_{G}\right) .
$$

Consequently, for anti-symmetric tensor products one has:

$$
\begin{aligned}
& \operatorname{ch}_{[\mathbf{R}]_{\mathbf{2}}^{-}}\left(A_{G}\right)=\frac{1}{2}\left(\operatorname{ch}_{\mathbf{R}}\left(A_{G}\right)^{2}-\operatorname{ch}_{\mathbf{R}}\left(2 A_{G}\right)\right), \\
& \operatorname{ch}_{[\mathbf{R}]_{\mathbf{3}}^{-}}\left(A_{G}\right)=\frac{1}{6}\left(\operatorname{ch}_{\mathbf{R}}\left(A_{G}\right)^{3}-3 \operatorname{ch}_{\mathbf{R}}\left(A_{G}\right) \operatorname{ch}_{\mathbf{R}}\left(2 A_{G}\right)+2 \operatorname{ch}_{\mathbf{R}}\left(3 A_{G}\right)\right),
\end{aligned}
$$

We denote by

$$
\operatorname{tr}\left(A_{\mathrm{SU}}\right)=\operatorname{tr}_{\mathbf{N}}\left(A_{\mathrm{SU}}\right)
$$

the trace as if it is the trace over the fundamental representation of an SU group, i.e. the vector representation $\mathbf{N}$ of the $\mathrm{SU}(N)$ group. Similarly, we define for the trace over the fundamental (vector) representations $\mathbf{2} \mathbf{N}$ of $\mathrm{SO}(2 N)$ and $\mathrm{Sp}(2 N)$,

$$
\operatorname{TR}\left(A_{\mathrm{SO}}\right)=\operatorname{tr}_{2 \mathbf{N}}\left(A_{\mathrm{SO}}\right), \quad \operatorname{TR}\left(A_{\mathrm{Sp}}\right)=\operatorname{tr}_{\mathbf{2 N}}\left(A_{\mathrm{Sp}}\right),
$$

For SO- and Sp-groups this means that

$$
\operatorname{TR}\left(A_{\mathrm{SU}}\right)=\operatorname{tr}_{\mathbf{2 N}}\left(A_{\mathrm{SU}}\right)=2 \operatorname{tr}_{\mathbf{N}}\left(A_{\mathrm{SU}}\right)=2 \operatorname{tr}\left(A_{\mathrm{SU}}\right),
$$




\begin{tabular}{|c|c|c|c|c|}
\hline \multicolumn{5}{|c|}{$\mathrm{SU}(N)$ representations } \\
Name & $\mathbf{R}$ & Weights $p$ & $|\mathbf{R}|$ & $\ell(\mathbf{R})$ \\
\hline Vector & $\mathbf{F}=\mathbf{N}$ & $\left(\underline{1,0^{N-1}}\right)$ & $N$ & 1 \\
Adjoint & $\mathbf{A d}$ & $\left(\frac{ \pm 1^{2}, 0^{N-2}}{\left(1^{2}, 0^{N-2}\right.}\right)$ & $N^{2}-1$ & $\frac{1}{2} N(N-1)$ \\
Rank-2 tensor & {$[\mathbf{N}]_{\mathbf{2}}^{-}$} & $(N)$ & $N-2$ \\
Rank-3 tensor & {$[\mathbf{N}]_{\mathbf{3}}^{-}$} & $\left(1^{3}, 0^{N-3}\right)$ & $\frac{1}{6} N(N-1)(N-2)$ & $\frac{1}{2}(N-2)(N-3)$ \\
$\vdots$ & $\vdots$ & $\vdots$ & $\vdots$ & $\vdots$ \\
\hline
\end{tabular}

Table 7. Some representations of $\mathrm{SU}(N)$ and their weights are indicated.

\begin{tabular}{|c|c|c|c|c|}
\hline \multicolumn{5}{|c|}{$\mathrm{SO}(2 N)$ representations } \\
Name & $\mathbf{R}$ & Weights $p$ & $|\mathbf{R}|$ & $\ell(\mathbf{R})$ \\
\hline Vector & $\mathbf{2 N}$ & $\left( \pm 1,0^{N-1}\right)$ & $2 N$ & 2 \\
Adjoint & $\mathbf{A d}$ & $\frac{\left( \pm 1^{2}, 0^{N-2}\right)}{\left( \pm \frac{1}{2}^{N}\right)}$ & $N(2 N-1)$ & $4(N-1)$ \\
Spinor $_{ \pm}$ & $\mathbf{2}_{ \pm}^{\mathbf{N}-\mathbf{1}}$ & $2^{N-1}$ & $2^{N-3}$ \\
& & $\#(-)=$ even/odd & & \\
\hline
\end{tabular}

Table 8. Some representations of $\mathrm{SO}(2 N)$ and their weights are indicated.

since we have the branching $\mathbf{2} \mathbf{N} \rightarrow \mathbf{N}+\overline{\mathbf{N}}$ when we consider $A_{\mathrm{SU}}^{\dagger}=A_{\mathrm{SU}}$ in the $\mathrm{SU}(N)$ subalgebra of the $\mathrm{SO}(2 N)$ algebra. We often write traces of objects valued in a certain algebra as traced over another representation associated to a different algebra. In this case this should be understood as defining some useful notation, not literally as the trace written (as that would not necessarily make sense). For example, the l.h.s. of

$$
\operatorname{tr}\left(A_{\mathrm{SO}}\right)=\operatorname{tr}_{\mathbf{N}}\left(A_{\mathrm{SO}}\right):=\frac{1}{2} \operatorname{tr}_{\mathbf{2 N}}\left(A_{\mathrm{SO}}\right),
$$

does not make sense because the smallest representation of $\mathrm{SO}(2 N)$ is $\mathbf{2} \mathbf{N}$ and not the fundamental representation $N$ of the $\mathrm{SU}(N)$ group. Hence, here the l.h.s. is defined as the r.h.s. including the normalization factor $1 / 2$. Similar one often uses the trace of the adjoint of $\mathrm{E}_{8}$ written as the trace over the fundamental of $\mathrm{SO}(16)$ :

$$
\operatorname{TR}\left(A_{\mathrm{E}_{8}}\right)=\operatorname{tr}_{16}\left(A_{\mathrm{E}_{8}}\right):=\frac{1}{30} \operatorname{tr}_{248}\left(A_{\mathrm{E}_{8}}\right) .
$$

We reserve the notation Tr to denote the trace over the full fermionic spectrum. For the supersymmetric $\mathrm{E}_{8} \times \mathrm{E}_{8}$ and $\mathrm{SO}(32)$ theories, this is then the trace in the adjoint of the respective gauge group.

The quadratic Casimir operator of the algebra is given by

$$
C_{G}=\sum_{A}\left(T_{A} T_{A}\right)_{G}
$$


Denoting the eigenvalue of this Casimir evaluated on a representation $\mathbf{R}$ by $C(\mathbf{R})$, we have

$$
C(\mathbf{R})|\mathbf{R}|=\sum_{A} \operatorname{tr}_{\mathbf{R}}\left(T_{A} T_{A}\right)_{G}
$$

This means that

$$
\operatorname{tr}_{\mathbf{N}}\left(T_{A} T_{B}\right)_{\mathrm{SU}}=\delta_{A B}, \quad \operatorname{tr}_{\mathbf{N}}(C)=|\mathbf{A d}|
$$

The index $\ell(\mathbf{R})$ of a representation $\mathbf{R}$ of a given group $G$ is defined as

$$
\operatorname{tr}_{\mathbf{R}}\left(T_{A} T_{B}\right)=\ell(\mathbf{R}) \operatorname{tr}_{\mathbf{N}}\left(T_{A} T_{B}\right) .
$$

By setting $B=A$ and summing over $A$, we obtain the trace (A.13) on the right-hand side. Because of the weights of the spinor representation $\mathbf{2}_{+}^{\mathbf{N}-\mathbf{1}}$, it branches into a sum of even rank anti-symmetric tensor representations $[\mathbf{N}]_{\mathbf{2} \mathbf{k}}^{-}$. Using this one can determine the index of the spinor representation if the indices of the anti-symmetric tensor representations of the SU group are known. The resulting values for some representations indices of SU and SO-groups can be found in tables 7 and 8 .

For quadratic traces of two Cartan generators in the fundamental representations of the SU and SO groups we find:

$$
\operatorname{tr}_{\mathbf{N}}\left(H_{I} H_{J}\right)=\delta_{I J}, \quad \operatorname{tr}_{\mathbf{2 N}}\left(H_{I} H_{J}\right)=2 \delta_{I J},
$$

respectively, and similarly for quartic traces:

$$
\operatorname{tr}_{\mathbf{N}}\left(H_{I} H_{J} H_{K} H_{L}\right)=\delta_{I J K L}, \quad \operatorname{tr}_{\mathbf{2 N}}\left(H_{I} H_{J} H_{K} H_{L}\right)=2 \delta_{I J K L},
$$

where $\delta_{I J K L}=1$ when all indices are equal and zero otherwise. We should stress that these trace identities only hold when traced over Cartan generators as stated here; generic quartic traces are more complicated.

\section{B Line bundle description as $\mathrm{S}\left(\mathrm{U}(1)^{n+1}\right)$ bundles}

The authors of some of the literature on line bundles on Calabi-Yau manifolds [64, 65, 99] use a different parameterization for the embedding of the structure group of the vector bundle into the primordial gauge group. We briefly review this parameterization in order to facilitate contact with our description. A vector bundle $\mathcal{V}$ with structure group $\mathrm{S}\left(\mathrm{U}(1)^{n+1}\right)$ can be obtained as a direct sum of line bundles

$$
\mathcal{V}=\bigoplus_{a=1}^{n+1} \mathcal{O}\left(k_{1}^{(a)}, \ldots, k_{h_{11}}^{(a)}\right)
$$

labeled by $n h_{11}$ integers $k_{i}^{(a)}$. This leads to a gauge flux that can be represented as

$$
\frac{\mathcal{F}}{2 \pi}=k_{i}^{(a)} D_{i} H_{(a)},
$$

where $H_{(a)}$ are the $n+1 \mathrm{U}(1)$ generators of the Cartan of $\mathrm{U}(\mathrm{n}+1)$. In particular, $k^{(a)}$ can be identified with the charge of the $a$-th 10-plet. Here we have expanded the first Chern classes $c_{1}\left(\mathcal{O}\left(k_{1}^{(a)}, \ldots k_{h_{11}}^{(a)}\right)=k_{i}^{(a)} \widehat{D}_{i}\right.$ associated to the divisors $D_{i}$. In order to ensure that we have an $\mathrm{S}\left(\mathrm{U}(1)^{n+1}\right)$ and not a $\mathrm{U}(1)^{n+1}$ structure group we require that $c_{1}(\mathcal{V})=0$, i.e. the corresponding gauge flux is traceless: $k_{i}^{(n+1)}=-k_{i}^{(1)}-\ldots-k_{i}^{(n)}$. 


\section{B.1 A line bundle vector representation of $\mathrm{S}\left(\mathrm{U}(1)^{5}\right)$ bundles}

In the literature mostly $\mathrm{S}\left(\mathrm{U}(1)^{5}\right) \supset \mathrm{E}_{8}$ bundles are discussed. To translate such bundle backgrounds into the language used in this work we observe the following: the $\mathrm{E}_{8}$ gauge group will contain an unbroken $\mathrm{SU}(5)$ group if we choose ${ }^{4}$ (up to overall permutations)

$$
V_{i}=\left(a_{i}^{5}, b_{i}, c_{i}, d_{i}\right),
$$

provided that the coefficients $a_{i} \neq 0, b_{i}, c_{i}, d_{i}$ are sufficiently generic, i.e. no entries are equal or opposite and the sums of all entries mod two does not vanish. Using this choice, all unbroken $\mathrm{SU}(5)$ roots, $\left(-1,1,0^{3}, 0^{3}\right)\left(0^{8}\right)$, are vectorial. In the non-supersymmetric case, this ansatz leads to the breaking of one of the $\mathrm{SO}(16)$ factors to $\mathrm{SU}(5)$.

To identify the integers $k_{i}^{(a)}$ with the quantities appearing in the parameterization $V_{i}$ given in (B.3) we compare the value of the charges of the 10-plets of $\mathrm{SU}(5)$ in both descriptions. The motivation to use the 10-plets for this matching is given by the branching the adjoint of $\mathrm{E}_{8}$

$$
248 \rightarrow(24,1)+(1,24)+(10,5)+(\overline{10}, \overline{5})+(5, \overline{10})+(\overline{5}, 10)
$$

under $\mathrm{E}_{8} \rightarrow \mathrm{SU}(5) \times \mathrm{SU}(5)$. Hence we see the that $\mathrm{U}(1)^{5}$ charges under the gauge fluxes supported on the divisor $\widehat{D}_{i}$ of the $\mathbf{5}$-components are simply $k_{(a)}^{i}$. Using this we obtain the

\begin{tabular}{|c|c|c|}
\hline 10-plets & $V_{i}$-charges & $k_{i}$-charges \\
\hline$\left(-\frac{1}{2}^{3}, \frac{1}{2}^{2},-\frac{1}{2}, \frac{1}{2}, \frac{1}{2}\right)$ & $-\frac{a_{i}}{2}-\frac{b_{i}}{2}+\frac{c_{i}}{2}+\frac{d_{i}}{2}$ & $k_{i}^{(1)}$ \\
\hline$\left(-\frac{1}{2}^{3}, \frac{1}{2}^{2}, \frac{1}{2},-\frac{1}{2}, \frac{1}{2}\right)$ & $-\frac{a_{i}}{2}+\frac{b_{i}}{2}-\frac{c_{i}}{2}+\frac{d_{i}}{2}$ & $k_{i}^{(2)}$ \\
\hline$\left(-\frac{1}{2}^{3}, \frac{1}{2}^{2}, \frac{1}{2}, \frac{1}{2},-\frac{1}{2}\right)$ & $-\frac{a_{i}}{2}+\frac{b_{i}}{2}+\frac{c_{i}}{2}-\frac{d_{i}}{2}$ & $k_{i}^{(3)}$ \\
\hline$\left(-\frac{1}{2}^{3}, \frac{1}{2}^{2},-\frac{1}{2},-\frac{1}{2},-\frac{1}{2}\right)$ & $-\frac{a_{i}}{2}-\frac{b_{i}}{2}-\frac{c_{i}}{2}-\frac{d_{i}}{2}$ & $k_{i}^{(4)}$ \\
\hline$\left(1^{2}, 0^{3}, 0^{3}\right)$ & & $k_{i}^{(5)}$ \\
\hline
\end{tabular}
following relation between the $\mathrm{U}(1)$ bundle charges in both languages:

Note that this identification automatically builds in the constraint on the sum, $\sum_{a} k_{i}^{(a)}=0$. Solving for the quantities in (B.3) we find

$$
\begin{array}{ll}
a_{i}=-\frac{1}{2}\left(k_{i}^{(1)}+k_{i}^{(2)}+k_{i}^{(3)}+k_{i}^{(4)}\right), & b_{i}=-\frac{1}{2}\left(k_{i}^{(1)}-k_{i}^{(2)}-k_{i}^{(3)}+k_{i}^{(4)}\right), \\
c_{i}=-\frac{1}{2}\left(-k_{i}^{(1)}+k_{i}^{(2)}-k_{i}^{(3)}+k_{i}^{(4)}\right), & d_{i}=-\frac{1}{2}\left(-k_{i}^{(1)}-k_{i}^{(2)}+k_{i}^{(3)}+k_{i}^{(4)}\right) .
\end{array}
$$

This shows in particular that when the sum of $k_{i}^{(1)}+\ldots+k_{i}^{(4)}$ is even (odd), we obtain the vectorial (spinorial) $\mathrm{E}_{8}$ weights. These identifications should be read modulo permutations.

\section{B.2 A line bundle vector representation of $\mathrm{S}\left(\mathrm{U}(1)^{6}\right)$ bundles}

Similarly we can identify other line bundle backgrounds in both descriptions. As an example we consider $\mathrm{S}\left(\mathrm{U}(1)^{6}\right)$ bundles with five independent bundle entries $k_{(a)}^{i}, a=1, \ldots, 5$. In this case the unbroken gauge group is generically $\mathrm{SU}(4)$, hence we consider the branching

$$
248 \rightarrow(15,1)+(1,45)+(4,16)+(\overline{4}, \overline{16})+(6,10)
$$

\footnotetext{
${ }^{4}$ Other parameterizations corresponding to different embeddings in the $\mathrm{E}_{8}$ are also possible.
} 
of $\mathrm{E}_{8} \rightarrow \mathrm{SU}(4) \times \mathrm{SO}(10)$. The anti-symmetric tensor 6 can now be used to identify the translation uniquely. It is paired with the $\mathbf{1 0}$-plet, which branches as $\mathbf{1 0} \rightarrow \mathbf{5}+\overline{\mathbf{5}}$ under $\mathrm{SO}(10) \rightarrow \mathrm{U}(5)$. Hence the five entries $k_{(a)}^{i}$ can be identified with the $\mathrm{U}(1)$ charges of the five components of the 5-plet in this branching. To realize this using the bundle vectors employed in this work, we take (up to overall permutations)

$$
V_{i}=\left(a_{i}^{4}, b_{i}, c_{i}, d_{i}, e_{i}\right) .
$$

Matching the 6-plet charges, we find:

\begin{tabular}{|c|c|c|}
\hline 6-plets & $V_{i}$-charges & $k_{i}$-charges \\
\hline$\left(-\frac{1}{2}^{2}, \frac{1}{2}^{2}, \frac{1}{2}, \frac{1}{2}, \frac{1}{2}, \frac{1}{2}\right)$ & $\frac{b_{i}}{2}+\frac{c_{i}}{2}+\frac{d_{i}}{2}+\frac{e_{i}}{2}$ & $k_{i}^{(1)}$ \\
\hline$\left(-\frac{1}{2}^{2}, \frac{1}{2}^{2},-\frac{1}{2},-\frac{1}{2}, \frac{1}{2}, \frac{1}{2}\right)$ & $-\frac{b_{i}}{2}-\frac{c_{i}}{2}+\frac{d_{i}}{2}+\frac{e_{i}}{2}$ & $k_{i}^{(2)}$ \\
\hline$\left(\overline{-\frac{1}{2}^{2}, \frac{1}{2}^{2}},-\frac{1}{2}, \frac{1}{2},-\frac{1}{2}, \frac{1}{2}\right)$ & $-\frac{b_{i}}{2}+\frac{c_{i}}{2}-\frac{d_{i}}{2}+\frac{e_{i}}{2}$ & $k_{i}^{(3)}$ \\
\hline$\left(-\frac{1}{2}^{2}, \frac{1}{2}^{2},-\frac{1}{2}, \frac{1}{2}, \frac{1}{2},-\frac{1}{2}\right)$ & $-\frac{b_{i}}{2}+\frac{c_{i}}{2}+\frac{d_{i}}{2}-\frac{e_{i}}{2}$ & $k_{i}^{(4)}$ \\
\hline$\left(-1^{2}, 0^{2}, 0^{4}\right)$ & $-2 a_{i}$ & $k_{i}^{(5)}$ \\
\hline
\end{tabular}

Open Access. This article is distributed under the terms of the Creative Commons Attribution License (CC-BY 4.0), which permits any use, distribution and reproduction in any medium, provided the original author(s) and source are credited.

\section{References}

[1] L.J. Dixon and J.A. Harvey, String theories in ten-dimensions without space-time supersymmetry, Nucl. Phys. B 274 (1986) 93 [INSPIRE].

[2] L.J. Dixon, J.A. Harvey, C. Vafa and E. Witten, Strings on orbifolds. 2., Nucl. Phys. B 274 (1986) 285 [inSPIRE].

[3] L. Álvarez-Gaumé, P.H. Ginsparg, G.W. Moore and C. Vafa, $A n O(16) \times O(16)$ heterotic string, Phys. Lett. B 171 (1986) 155 [INSPIRE].

[4] R. Rohm, Spontaneous supersymmetry breaking in supersymmetric string theories, Nucl. Phys. B 237 (1984) 553 [inSPIRE].

[5] C. Kounnas and B. Rostand, Coordinate Dependent Compactifications and Discrete Symmetries, Nucl. Phys. B 341 (1990) 641 [InSPIRE].

[6] D. Kutasov and N. Seiberg, Number of degrees of freedom, density of states and tachyons in string theory and CFT, Nucl. Phys. B 358 (1991) 600 [INSPIRE].

[7] J.A. Harvey, String duality and nonsupersymmetric strings, Phys. Rev. D 59 (1999) 026002 [hep-th/9807213] [INSPIRE].

[8] V.P. Nair, A.D. Shapere, A. Strominger and F. Wilczek, Compactification of the twisted heterotic string, Nucl. Phys. B 287 (1987) 402 [INSPIRE].

[9] P.H. Ginsparg and C. Vafa, Toroidal compactification of nonsupersymmetric heterotic strings, Nucl. Phys. B 289 (1987) 414 [InSPIRE].

[10] W. Lerche, D. Lüst and A.N. Schellekens, Ten-dimensional heterotic strings from Niemeier lattices, Phys. Lett. B 181 (1986) 71 [INSPIRE]. 
[11] W. Lerche, D. Lüst and A.N. Schellekens, Chiral four-dimensional heterotic strings from selfdual lattices, Nucl. Phys. B 287 (1987) 477 [INSPIRE].

[12] L.J. Dixon, J.A. Harvey, C. Vafa and E. Witten, Strings on orbifolds, Nucl. Phys. B 261 (1985) 678 [inSPIRE].

[13] L.E. Ibáñez, H.P. Nilles and F. Quevedo, Orbifolds and Wilson lines, Phys. Lett. B 187 (1987) 25 [InSPIRE].

[14] L.E. Ibáñez, J. Mas, H.-P. Nilles and F. Quevedo, Heterotic strings in symmetric and asymmetric orbifold backgrounds, Nucl. Phys. B 301 (1988) 157 [INSPIRE].

[15] T.R. Taylor, Model building on asymmetric Z(3) orbifolds: Nonsupersymmetric models, Nucl. Phys. B 303 (1988) 543 [inSPIRE].

[16] A. Toon, Nonsupersymmetric Z(4) orbifolds and Atkin-Lehner symmetry, Phys. Lett. B 243 (1990) 68 [InSPIRE].

[17] T. Sasada, Asymmetric orbifold models of nonsupersymmetric heterotic strings, Prog. Theor. Phys. 95 (1996) 249 [hep-th/9508098] [InSPIRE].

[18] A. Font and A. Hernández, Nonsupersymmetric orbifolds, Nucl. Phys. B 634 (2002) 51 [hep-th/0202057] [INSPIRE].

[19] H. Kawai, D.C. Lewellen and S.H.H. Tye, Classification of closed fermionic string models, Phys. Rev. D 34 (1986) 3794 [InSPIRE].

[20] I. Antoniadis, C.P. Bachas and C. Kounnas, Four-Dimensional Superstrings, Nucl. Phys. B 289 (1987) 87 [inSPIRE].

[21] I. Antoniadis and C. Bachas, $4-D$ Fermionic Superstrings with Arbitrary Twists, Nucl. Phys. B 298 (1988) 586 [inSPIRE].

[22] K.R. Dienes, Modular invariance, finiteness and misaligned supersymmetry: New constraints on the numbers of physical string states, Nucl. Phys. B 429 (1994) 533 [hep-th/9402006] [INSPIRE].

[23] J.D. Blum and K.R. Dienes, Duality without supersymmetry: The case of the $\mathrm{SO}(16) \times \mathrm{SO}(16)$ string, Phys. Lett. B 414 (1997) 260 [hep-th/9707148] [INSPIRE].

[24] G. Shiu and S.H.H. Tye, Bose-Fermi degeneracy and duality in nonsupersymmetric strings, Nucl. Phys. B 542 (1999) 45 [hep-th/9808095] [INSPIRE].

[25] K.R. Dienes, Statistics on the heterotic landscape: Gauge groups and cosmological constants of four-dimensional heterotic strings, Phys. Rev. D 73 (2006) 106010 [hep-th/0602286] [INSPIRE].

[26] A.E. Faraggi and M. Tsulaia, On the low energy spectra of the nonsupersymmetric heterotic string theories, Eur. Phys. J. C 54 (2008) 495 [arXiv:0706.1649] [InSPIRE].

[27] C. Angelantonj, I. Antoniadis and K. Forger, Nonsupersymmetric type-I strings with zero vacuum energy, Nucl. Phys. B 555 (1999) 116 [hep-th/9904092] [INSPIRE].

[28] C. Angelantonj, I. Antoniadis, G. D'Appollonio, E. Dudas and A. Sagnotti, Type I vacua with brane supersymmetry breaking, Nucl. Phys. B 572 (2000) 36 [hep-th/9911081] [INSPIRE].

[29] C. Angelantonj and E. Dudas, Metastable string vacua, Phys. Lett. B 651 (2007) 239 [arXiv: 0704 . 2553] [INSPIRE].

[30] A. Sagnotti, Some properties of open string theories, hep-th/9509080 [INSPIRE]. 
[31] A. Sagnotti, Surprises in open string perturbation theory, Nucl. Phys. Proc. Suppl. 56B (1997) 332 [hep-th/9702093] [InSPIRE].

[32] C. Angelantonj, Nontachyonic open descendants of the $0 B$ string theory, Phys. Lett. B 444 (1998) 309 [hep-th/9810214] [INSPIRE].

[33] I. Antoniadis, E. Dudas and A. Sagnotti, Brane supersymmetry breaking, Phys. Lett. B 464 (1999) 38 [hep-th/9908023] [INSPIRE].

[34] S. Sugimoto, Anomaly cancellations in type-I D-9-DD-9 system and the USp(32) string theory, Prog. Theor. Phys. 102 (1999) 685 [hep-th/9905159] [INSPIRE].

[35] R. Blumenhagen, A. Font and D. Lüst, Tachyon free orientifolds of type OB strings in various dimensions, Nucl. Phys. B 558 (1999) 159 [hep-th/9904069] [INSPIRE].

[36] G. Aldazabal and A.M. Uranga, Tachyon free nonsupersymmetric type IIB orientifolds via brane-anti-brane systems, JHEP 10 (1999) 024 [hep-th/9908072] [INSPIRE].

[37] G. Aldazabal, L.E. Ibáñez and F. Quevedo, Standard-like models with broken supersymmetry from type-I string vacua, JHEP 01 (2000) 031 [hep-th/9909172] [INSPIRE].

[38] E. Dudas and J. Mourad, Brane solutions in strings with broken supersymmetry and dilaton tadpoles, Phys. Lett. B 486 (2000) 172 [hep-th/0004165] [inSPIRE].

[39] S. Moriyama, USp(32) string as spontaneously supersymmetry broken theory, Phys. Lett. B 522 (2001) 177 [hep-th/0107203] [INSPIRE].

[40] E. Dudas, J. Mourad and C. Timirgaziu, Time and space dependent backgrounds from nonsupersymmetric strings, Nucl. Phys. B 660 (2003) 3 [hep-th/0209176] [INSPIRE].

[41] B. Gato-Rivera and A.N. Schellekens, Non-supersymmetric Tachyon-free Type-II and Type-I Closed Strings from RCFT, Phys. Lett. B 656 (2007) 127 [arXiv: 0709.1426] [INSPIRE].

[42] B. Gato-Rivera and A.N. Schellekens, Non-supersymmetric orientifolds of Gepner models, Phys. Lett. B 671 (2009) 105 [arXiv:0810.2267] [InSPIRE].

[43] M. Blaszczyk, S. Groot Nibbelink, O. Loukas and S. Ramos-Sanchez, Non-supersymmetric heterotic model building, JHEP 10 (2014) 119 [arXiv:1407.6362] [INSPIRE].

[44] S.G. Nibbelink, Model building with the non-supersymmetric heterotic $\mathrm{SO}(16) \times \mathrm{SO}(16)$ string, J. Phys. Conf. Ser. 631 (2015) 012077 [arXiv: 1502.03604] [INSPIRE].

[45] A. Lukas, Z. Lalak and E.E. Svanes, Heterotic moduli stabilisation and non-supersymmetric vacua, JHEP 08 (2015) 020 [arXiv:1504.06978] [INSPIRE].

[46] S. Abel, K.R. Dienes and E. Mavroudi, Towards a nonsupersymmetric string phenomenology, Phys. Rev. D 91 (2015) 126014 [arXiv:1502.03087] [INSPIRE].

[47] J.M. Ashfaque, P. Athanasopoulos, A.E. Faraggi and H. Sonmez, Non-Tachyonic Semi-Realistic Non-Supersymmetric Heterotic String Vacua, arXiv:1506.03114 [InSPIRE].

[48] H. Itoyama and T.R. Taylor, Supersymmetry Restoration in the Compactified O(16) $\times$ O(16)-prime Heterotic String Theory, Phys. Lett. B 186 (1987) 129 [INSPIRE].

[49] K.R. Dienes, New string partition functions with vanishing cosmological constant, Phys. Rev. Lett. 65 (1990) 1979 [INSPIRE].

[50] A.E. Faraggi and M. Tsulaia, Interpolations Among NAHE-based Supersymmetric and Nonsupersymmetric String Vacua, Phys. Lett. B 683 (2010) 314 [arXiv:0911.5125] [INSPIRE]. 
[51] C. Angelantonj, I. Florakis and M. Tsulaia, Universality of Gauge Thresholds in Non-Supersymmetric Heterotic Vacua, Phys. Lett. B 736 (2014) 365 [arXiv:1407.8023] [INSPIRE].

[52] I. Florakis, Universality of radiative corrections to gauge couplings for strings with spontaneously broken supersymmetry, J. Phys. Conf. Ser. 631 (2015) 012079 [arXiv: 1502.07537] [INSPIRE].

[53] C.M. Hull and E. Witten, Supersymmetric $\sigma$-models and the Heterotic String, Phys. Lett. B 160 (1985) 398 [inSPIRE].

[54] C.M. Hull, Complex structures and isometries in the (2,0) supersymmetric nonlinear $\sigma$-model, Mod. Phys. Lett. A 5 (1990) 1793 [INSPIRE].

[55] R. Blumenhagen, G. Honecker and T. Weigand, Loop-corrected compactifications of the heterotic string with line bundles, JHEP 06 (2005) 020 [hep-th/0504232] [INSPIRE].

[56] R. Blumenhagen, G. Honecker and T. Weigand, Supersymmetric (non-)Abelian bundles in the Type I and $\mathrm{SO}(32)$ heterotic string, JHEP 08 (2005) 009 [hep-th/0507041] [INSPIRE].

[57] R. Blumenhagen, S. Moster and T. Weigand, Heterotic GUT and standard model vacua from simply connected Calabi-Yau manifolds, Nucl. Phys. B 751 (2006) 186 [hep-th/0603015] [INSPIRE].

[58] V. Braun, Y.-H. He, B.A. Ovrut and T. Pantev, A heterotic standard model, Phys. Lett. B 618 (2005) 252 [hep-th/0501070] [INSPIRE].

[59] V. Bouchard and R. Donagi, An SU(5) heterotic standard model, Phys. Lett. B 633 (2006) 783 [hep-th/0512149] [INSPIRE].

[60] L.B. Anderson, Y.-H. He and A. Lukas, Heterotic compactification, an algorithmic approach, JHEP 07 (2007) 049 [hep-th/0702210] [INSPIRE].

[61] S. Groot Nibbelink, M. Trapletti and M. Walter, Resolutions of $C^{n} / Z_{n}$ orbifolds, their $\mathrm{U}(1)$ bundles and applications to string model building, JHEP 03 (2007) 035 [hep-th/0701227] [INSPIRE].

[62] S. Groot Nibbelink, J. Held, F. Ruehle, M. Trapletti and P.K.S. Vaudrevange, Heterotic Z(6-II) MSSM orbifolds in blowup, JHEP 03 (2009) 005 [arXiv:0901.3059] [INSPIRE].

[63] M. Blaszczyk, S. Groot Nibbelink, F. Ruehle, M. Trapletti and P.K.S. Vaudrevange, Heterotic MSSM on a resolved orbifold, JHEP 09 (2010) 065 [arXiv: 1007. 0203] [INSPIRE].

[64] L.B. Anderson, J. Gray, A. Lukas and E. Palti, Two Hundred Heterotic Standard Models on Smooth Calabi-Yau Threefolds, Phys. Rev. D 84 (2011) 106005 [arXiv:1106.4804] [INSPIRE].

[65] L.B. Anderson, J. Gray, A. Lukas and E. Palti, Heterotic line bundle Standard Models, JHEP 06 (2012) 113 [arXiv:1202.1757] [INSPIRE].

[66] P. Candelas, G.T. Horowitz, A. Strominger and E. Witten, Vacuum configurations for superstrings, Nucl. Phys. B 258 (1985) 46 [InSPIRE].

[67] A. Strominger, Superstrings with torsion, Nucl. Phys. B 274 (1986) 253 [inSPIRE].

[68] P. Candelas, A.M. Dale, C.A. Lütken and R. Schimmrigk, Complete Intersection Calabi-Yau Manifolds, Nucl. Phys. B 298 (1988) 493 [INSPIRE].

[69] CALABI-YAU Home Page: A resource for information about Calabi-Yau manifolds, http://www.th.physik.uni-bonn.de/Supplements/cy.html (1996). 
[70] V. Braun, On Free Quotients of Complete Intersection Calabi-Yau Manifolds, JHEP 04 (2011) 005 [arXiv: 1003.3235] [INSPIRE].

[71] M.B. Green and J.H. Schwarz, Anomaly cancellation in supersymmetric D $=10$ gauge theory and superstring theory, Phys. Lett. B 149 (1984) 117 [INSPIRE].

[72] M.B. Green, J.H. Schwarz and P.C. West, Anomaly free chiral theories in six-dimensions, Nucl. Phys. B 254 (1985) 327 [inSPIRE].

[73] M.B. Green, J.H. Schwarz and E. Witten, Superstring theory. Vol. 2: Loop amplitudes, anomalies and phenomenology, Cambridge Monographs On Mathematical Physics, Cambridge University Press, Cambridge U.K. (1987).

[74] N. Marcus, A. Sagnotti and W. Siegel, Ten-dimensional Supersymmetric Yang-Mills Theory in Terms of Four-dimensional Superfields, Nucl. Phys. B 224 (1983) 159 [InSPIRE].

[75] N. Arkani-Hamed, T. Gregoire and J.G. Wacker, Higher dimensional supersymmetry in 4 -D superspace, JHEP 03 (2002) 055 [hep-th/0101233] [INSPIRE].

[76] G. Lopes Cardoso, G. Curio, G. Dall'Agata, D. Lüst, P. Manousselis and G. Zoupanos, Non-Kähler string backgrounds and their five torsion classes, Nucl. Phys. B 652 (2003) 5 [hep-th/0211118] [INSPIRE].

[77] K. Becker, M. Becker, K. Dasgupta and P.S. Green, Compactifications of heterotic theory on nonKähler complex manifolds. 1., JHEP 04 (2003) 007 [hep-th/0301161] [INSPIRE].

[78] J.P. Gauntlett, D. Martelli and D. Waldram, Superstrings with intrinsic torsion, Phys. Rev. D 69 (2004) 086002 [hep-th/0302158] [inSPIRE].

[79] K. Becker, M. Becker, P.S. Green, K. Dasgupta and E. Sharpe, Compactifications of heterotic strings on nonKähler complex manifolds. 2., Nucl. Phys. B 678 (2004) 19 [hep-th/0310058] [INSPIRE].

[80] K. Becker, M. Becker, J.-X. Fu, L.-S. Tseng and S.-T. Yau, Anomaly cancellation and smooth non-Kähler solutions in heterotic string theory, Nucl. Phys. B 751 (2006) 108 [hep-th/0604137] [INSPIRE].

[81] M. Fernandez, S. Ivanov, L. Ugarte and R. Villacampa, Non-Kähler Heterotic String Compactifications with non-zero fluxes and constant dilaton, Commun. Math. Phys. 288 (2009) 677 [arXiv: 0804.1648] [INSPIRE].

[82] M. Klaput, A. Lukas, C. Matti and E.E. Svanes, Moduli stabilising in heterotic nearly Káhler compactifications, JHEP 01 (2013) 015 [arXiv:1210.5933] [INSPIRE].

[83] S. Hosono, A. Klemm, S. Theisen and S.-T. Yau, Mirror symmetry, mirror map and applications to complete intersection Calabi-Yau spaces, Nucl. Phys. B 433 (1995) 501 [hep-th/9406055] [INSPIRE].

[84] A. Lukas and K.S. Stelle, Heterotic anomaly cancellation in five-dimensions, JHEP 01 (2000) 010 [hep-th/9911156] [INSPIRE].

[85] S. Groot Nibbelink, T.-W. Ha and M. Trapletti, Toric resolutions of heterotic orbifolds, Phys. Rev. D 77 (2008) 026002 [arXiv:0707.1597] [inSPIRE].

[86] S. Groot Nibbelink, D. Klevers, F. Ploger, M. Trapletti and P.K.S. Vaudrevange, Compact heterotic orbifolds in blow-up, JHEP 04 (2008) 060 [arXiv: 0802.2809] [INSPIRE].

[87] M. Nakahara, Geometry, topology and physics, Graduate Student Series in Physics, Adam Hilger, Bristol U.K. (1990). 
[88] G. Honecker, Massive U(1)s and heterotic five-branes on K3, Nucl. Phys. B 748 (2006) 126 [hep-th/0602101] [INSPIRE].

[89] R. Blumenhagen, B. Jurke, T. Rahn and H. Roschy, Cohomology of Line Bundles: A Computational Algorithm, J. Math. Phys. 51 (2010) 103525 [arXiv:1003.5217] [INSPIRE].

[90] cohomCalg package, High-performance line bundle cohomology computation based on [89], http://wwwth.mppmu.mpg.de/members/blumenha/cohomcalg/ (2010).

[91] E. Witten, Dimensional Reduction of Superstring Models, Phys. Lett. B 155 (1985) 151 [INSPIRE].

[92] J.P. Derendinger, L.E. Ibáñez and H.P. Nilles, On the Low-Energy Limit of Superstring Theories, Nucl. Phys. B 267 (1986) 365 [InSPIRE].

[93] S. Ferrara, C. Kounnas and M. Porrati, General Dimensional Reduction of Ten-Dimensional Supergravity and Superstring, Phys. Lett. B 181 (1986) 263 [InSPIRE].

[94] L.J. Dixon, V. Kaplunovsky and J. Louis, On Effective Field Theories Describing (2,2) Vacua of the Heterotic String, Nucl. Phys. B 329 (1990) 27 [InSPIRE].

[95] A. Strominger, Yukawa couplings in superstring compactification, Phys. Rev. Lett. 55 (1985) 2547 [INSPIRE].

[96] E. Witten, Symmetry Breaking Patterns in Superstring Models, Nucl. Phys. B 258 (1985) 75 [INSPIRE].

[97] K.-S. Choi, K. Hwang and J.E. Kim, Dynkin diagram strategy for orbifolding with Wilson lines, Nucl. Phys. B 662 (2003) 476 [hep-th/0304243] [INSPIRE].

[98] N. Seiberg and E. Witten, Comments on string dynamics in six-dimensions, Nucl. Phys. B 471 (1996) 121 [hep-th/9603003] [INSPIRE].

[99] L.B. Anderson, A. Constantin, J. Gray, A. Lukas and E. Palti, A comprehensive scan for heterotic SU(5) GUT models, JHEP 01 (2014) 047 [arXiv: 1307.4787] [INSPIRE]. 